COMMUNICATIONS IN

ANALYSIS AND GEOMETRY

Volume 4, Number 3, 495-524, 1996

\title{
Infinitesimal rigidity of higher rank lattice actions
}

\section{NANTIAN QIaN}

We establish the continuous and smooth infinitesimal rigidity of standard actions of higher rank lattices.

\section{Introduction and the Statement of Results.}

Let $\Gamma$ be a discrete group, $M$ a smooth compact manifold without boundary, and $\rho$ a smooth action of $\Gamma$ on $M$ (or equivalently, $\rho$ is a homomorphism from $\Gamma$ to $\operatorname{Diff}^{\infty}(M)$ ). Let $\epsilon>0$ and $\rho_{t}, 0 \leq t \leq \epsilon, \rho_{0}=\rho$, be a differentiable path of smooth actions; i.e., for every $\gamma \in \Gamma, t \mapsto \rho_{t}(\gamma)$ is a differentiable path in Diff ${ }^{\infty}(M)$. Observe that for all $\gamma_{1}, \gamma_{2} \in \Gamma, t \in[0, \epsilon]$, $\rho_{t}\left(\gamma_{1} \gamma_{2}\right)(x)=\rho_{t}\left(\gamma_{1}\right) \rho_{t}\left(\gamma_{2}\right)(x)$. We differentiate the equation with respect to $t$ at $t=0$ and for any $\gamma \in \Gamma$ and $x \in M$ denote $\beta(\gamma)(x):=\left.\frac{d}{d t}\right|_{t=0} \rho_{t}(\gamma)(x)$, $\alpha(\gamma)(x):=\beta(\gamma)\left(\rho_{0}\left(\gamma^{-1}\right) x\right)$, then

$$
\alpha\left(\gamma_{1} \gamma_{2}\right)=\alpha\left(\gamma_{1}\right)+\left(\rho_{0}\left(\gamma_{1}\right)\right)_{*} \alpha\left(\gamma_{2}\right),
$$

where for any diffeomorphism $f$ and vector field $v, f_{*} v$ is a new vector field, $x \mapsto D f\left(v\left(f^{-1} x\right)\right)$.

For each $\gamma \in \Gamma$, assume that $\rho_{t}$ is obtained by a differentiable path of conjugacy with $\rho_{0}$, or equivalently there exists a differentiable path $\phi_{t}$ starting at Id in $\operatorname{Diff}^{\infty}(M)$ such that $\rho_{t}(\gamma)(x)=\phi_{t} \circ \rho_{0}(\gamma) \circ \phi_{t}^{-1}(x)$. Differentiating the equation with respect to $t$ at $t=0$, we get

$$
\alpha(\gamma)=v-\left(\rho_{0}(\gamma)\right)_{*} v
$$

for a vector field $v(x)=\left.\frac{d}{d t}\right|_{t=0} \phi_{t}(x)$.

Recall that a given action $\rho$ of $\Gamma$ on $M$ is said to be deformation rigid if for any sufficiently small deformation $\rho_{t}, \rho_{t}$ is given by conjugacy; and it is said to be infinitesimal rigid if all maps from $\Gamma$ to the set of smooth vector fields satisfying (1) (such maps are called cocycles) also satisfy (2) (such maps are called coboundaries). (We omit the reference to the class of smoothness and the topology for simplicity, see $\S 2$ for detailed definition). It is natural to ask the connection between the two notions of rigidity. Although no result 
is established in either direction, one anticipates that they are equivalent in a suitable sense (see [1] for a partial result) for standard higher rank lattice actions.

We are interested in the infinitesimal rigidity of discrete group actions. While arbitrary actions of discrete group $\Gamma$ may not be infinitesimal rigidity, one anticipates that the standard actions of higher rank lattices are infinitesimal rigid. For higher rank lattices, three types of standard volume preserving actions of higher rank lattice $\Gamma$ on a compact smooth manifold $M$ have been described by Zimmer: (1) Isometric actions; (2) Left translations on compact quotients $H / \Lambda$ via homomorphism $\Gamma \rightarrow H$, where $H$ is a connected Lie group, $\Lambda \subset H$ is a cocompact lattice; (3) Affine actions on compact nilmanifolds. We call them type 1 , type 2 and type 3 standard actions respectively.

The first infinitesimal rigidity result was obtained by Zimmer (Theorems 4.6, 5.6 of [20]) for type 2 standard actions. Let $G$ be a connected semisimple Lie group with no simple factors locally isomorphic to $O(1, n)$ or $U(1, n), H$ a Lie group, $\Gamma \subset G$ a cocompact lattice, $\Lambda \subset H$ a cocompact lattice. Let $\Gamma$ act ergodically on compact manifold $H / \Lambda$ via a homomorphism $\pi: \Gamma \rightarrow H$. Then any $C^{\infty}$-cocycle is an $L^{2}$-coboundary (the action is then said to be $L^{2}$ infinitesimal rigid). If moreover $H$ is semisimple and either (a) $\pi(\Gamma)$ is dense in $H$ or (b) $H=H_{1} \times H_{2}$, and $\pi(\Gamma)$ projects densely into $H_{1}$ and trivially into $H_{2}$, then any $C^{\infty}$-cocycle is a $C^{\infty}$-coboundary (the action is then said to be $C^{\infty}$-infinitesimal rigid). Actually, Zimmer proved more about the $L^{2}$ infinitesimal rigidity (see Theorem 4.5 of [20]). Later on Lewis [9] obtained the $L^{2}$-infinitesimal rigidity for non-cocompact lattice actions under some technique conditions, and $C^{\infty}$-infinitesimal rigidity of standard actions of $S L(n, \mathbb{Z})$ on torus $\mathbb{T}^{n}$ for $n \geq 7$. The overall structure of their proofs is that they show any $C^{\infty}$-cocycle is $L^{2}$-coboundary by Hodge theory on a suitable foliated manifold, and then show the regularity of the coboundary by Sobolev space techniques. We also mention that Lubotzky and Zimmer [13] obtained that for irreducible lattices $\Gamma$ in the product of a noncompact simple group with a semisimple Kazhdan group, isometric ergodic actions on compact manifolds by $\Gamma$ are $C^{\infty}$-infinitesimal rigid.

While we will refrain ourselves from discussing the detailed arguments of [20], [9], it is worth mentioning that Lewis used the hyperbolicity of the standard action of $S L(n, \mathbb{Z})$ on torus $\mathbb{T}^{n}$ to obtain the regularity result. Hurder [5] noticed that if the action $\rho$ of $\Gamma$ on $M$ is Anosov (i.e., there exists $\gamma \in \Gamma$ such that $\rho(\gamma)$ is Anosov diffeomorphism) with dense periodic points ( $p \in M$ is said to be periodic if the orbit of $p$ under $\rho$ is finite), and $\Gamma$ satisfies SVC (see $\S 2$ for definition), then any $C^{0}$-cocycle is $C^{0}$ - 
coboundary, and if $\rho$ is maximal Cartan (see [5] for definition), then $\rho$ is $C^{\infty}$-infinitesimal rigid. Hurder's argument used the hyperbolicity and the density of the periodic points of the action to obtain the $C^{0}$-infinitesimal rigidity, the regularity result is an application of the Livsic theorem for cocycle over Anosov diffeomorphism [10]. We remark that Hurder does not require the actions to be standard to obtain the continuous infinitesimal rigidity.

We extend Hurder's $C^{0}$-infinitesimal rigidity result to partially hyperbolic actions (§3). The regularity from $L^{2}$-infinitesimal rigidity to $C^{0}$ infinitesimal rigidity and then to $C^{\infty}$-infinitesimal rigidity is also established under some (very relaxed) technique conditions. The main new ingredient is an observation that an $\mathbb{R}^{n}$-valued function $f$ on $M$ is regular iff the projections of $f$ to sufficiently many subspaces are regular (see $\$ 2.5$ for exact results).

Theorem 1.1. Let $\Gamma$ be a discrete, Kazhdan, SVC group, $\rho$ a tangentially flat action of $\Gamma$ on a compact manifold with dense periodic points associated with a homomorphism $\pi: \Gamma \rightarrow G L(n, \mathbb{R})$. Then

1. $\rho$ is $C^{0}$-infinitesimal rigid if $\pi$ has no compact part (Proposition 3.2);

2. $\rho$ is $L^{2}$-infinitesimal rigid if $\pi$ decomposes as direct sum $\pi_{1}+\pi_{2}$, where $\pi_{1}$ has no compact part and $\pi_{2}$ has only compact part (Proposition 3.5).

For the definitions of tangential flatness and a homomorphism having no compact part, see $\S 2.2$ (D9-10).

Theorem 1.2. Let $\Gamma$ be a discrete, Kazhdan, SVC group, $M$ a torus or a nilmanifold, $\rho$ an action of $\Gamma$ on $M$ by automorphisms. Assume that the homomorphism $\pi$ corresponding to the tangent map has no compact part, is of almost Lyapunov multiplicity free type (for the definition, see §2.3), and the adjoint representation $A d \circ \pi$ of $\Gamma$ on $g l(n, \mathbb{R})$ is completely reducible.

1. Assume that $\rho$ is Anosov. Then $\rho$ is $C^{\infty}$-infinitesimal rigid (Theorem 4.2 in $\S 4)$.

2. Assume that $M$ is a torus. Then $\rho$ is $C^{\infty}$-infinitesimal rigid (Theorem 4.6 in $\S 4)$.

Corollary 1.3. Let $G$ be a connected, $\mathbb{R}$-split linear group without compact factor and finite center, $\Gamma$ a lattice in $G, \pi: G \rightarrow G L(n, \mathbb{R})$ a continuous homomorphism. If either (1) $\Gamma$ is irreducible and the real rank of $G$ is at 
least 2 or (2) the real rank of each simple factor of $G$ is at least 2 , and for any irreducible constituent $\pi^{\prime}$ of $\pi$, there exists a multiplicity free weight $\lambda^{\prime} \neq 0$ (i.e., any weight of $\pi$ is not proportional to $\lambda^{\prime}$ ).

1. Assume that $\rho$ is an Anosov action of $\Gamma$ on a torus or a nilmanifold by automorphisms associated with $\left.\pi\right|_{\Gamma}$. Then $\rho$ is $C^{\infty}$-infinitesimal rigid.

2. Assume that $\rho$ is an action of $\Gamma$ on a torus by automorphisms associated with $\left.\pi\right|_{\Gamma}$. Then $\rho$ is $C^{\infty}$-infinitesimal rigid.

Proof. It follows from Proposition 2.2 and Theorem 1.2.

Theorem 1.4. Let $H$ be a finite product of connected non-compact simple Lie groups $H_{i}$ with finite center, $\mathfrak{h}=\oplus \mathfrak{h}_{i}$ the decomposition of the Lie algebra of $H, \Lambda$ an irreducible cocompact lattice in $H$. Let $G$ be a connected semisimple Lie group with no simple factors locally isomorphic to $O(1, n)$ or $U(1, n), \Gamma \subset G$ a cocompact lattice. Let $\rho$ be an action of $\Gamma$ on $H / \Lambda$ via homomorphism $h: \Gamma \rightarrow H$. Assume that there exists $\gamma \in \Gamma$ such that $A d_{H} \circ h(\gamma)$ is partially hyperbolic, and the eigenspace $V_{1} \subset \mathfrak{h}$ corresponding to eigenvalue 1 of $A d_{H} \circ h(\gamma)$ satisfies $V_{1} \cap \mathfrak{h}_{i} \neq \mathfrak{h}_{i}, \neq\{0\}$. Assume also that $A d_{H} \circ h: \Gamma \rightarrow G L(\mathfrak{h})$ has no compact part, and the only non-trivial irreducible invariant spaces of it are $\mathfrak{h}_{i}$. Then $\rho$ is $C^{\infty}$-infinitesimal rigid (Theorems 4.11, 4.7 in §4).

Corollary 1.5. Assume the notation as in Theorem 1.4. If $h(\Gamma)$ is Zariski dense in $H$, then the standard action of $\Gamma$ on $H / \Lambda$ by left translation via $h$ is $C^{\infty}$-infinitesimal rigid.

Proof. The existence of an $\mathbb{R}$-regular element $s \in h(\Gamma)$ is established by Benoist-Labourie, Prasad (See for example [16]). Then $s$ is in a Cartan subgroup $C$ of $H$. Let $\mathfrak{c}$ be the Cartan subalgebra corresponding to $C$. Then $A d_{H} \circ h(s)$ has eigenspace $V_{1}=\mathfrak{c}$ corresponding to eigenvalue 1 [17].

Then we have $V_{1} \cap \mathfrak{h}_{i} \neq 0, \mathfrak{h}_{i}$. Moreover, $A d_{H} \circ h$ has no compact part, and the only non-trivial irreducible invariant spaces of it are $\mathfrak{h}_{i}$. So $\rho$ is $C^{\infty}$-infinitesimal rigid.

Corollary 1.6. Let $G, H$ be as in Theorem 1.4, and $H=G$. For any irreducible cocompact lattice $\Lambda \subset G$, cocompact lattice $\Gamma \subset G$ the standard action of $\Gamma$ on $G / \Lambda$ by left translation is $C^{\infty}$-infinitesimal rigid. 
Proof. It is a special case of Corollary 1.5 by Borel density theorem.

We thank D. Burde and R. Howe for helpful conversations.

\section{Preliminaries.}

For the convenience of the reader, we collect notations, definitions and preliminary results in this section. We mention that the results in $\S 2.5$ are important for the rest of the paper.

\subsection{Notations.}

We always assume the following unless otherwise specified.

1. (N1) $\Gamma$ is a discrete subgroup;

2. (N2) $M$ is a smooth compact manifold with dimension $n$;

3. (N3) $\rho$ is a $C^{\infty}$-action of $\Gamma$ on $M$;

4. (N4) $\Omega(\rho)$ the set of periodic points, $\Omega(\rho)=\{p \in M: \rho(\Gamma) p$ is finite $\}$;

5. (N5) $E$ is a $C^{\infty}$-vector bundle over $M$;

6. (N6) $\tilde{\rho}$ is a $C^{\infty}$-action of $\Gamma$ on $E$ by $C^{\infty}$-bundle automorphism, $\tilde{\rho}$ covers $\rho$;

7. (N7) $\mathcal{X}^{r}(E), \mathcal{X}^{b d d}(E), \mathcal{X}^{L^{2}}(E)$ are the sets of all $C^{r}$, bounded, $L^{2}$ sections of $E \rightarrow M$, respectively;

8. (N8) $(\tilde{\rho})_{*}$ is the action of $\Gamma$ on $\mathcal{X}^{r}(E), \mathcal{X}^{b d d}(E), \mathcal{X}^{L^{2}}(E),(\gamma, v(\cdot)) \mapsto$ $\tilde{\rho}(\gamma) v\left(\rho\left(\gamma^{-1}\right)(\cdot)\right)$ for all elements $\gamma \in \Gamma$ and sections $v$.

\subsection{Definitions.}

For the convenience of the reader, we put all definitions here for easy reference. All of them are standard except $(\mathrm{D} 9,10)$.

1. (D1) (1-cocycle, 1-coboundary and 1-cohomology group over a representation). Let $\pi$ be a representation of a group $H$ on a vector space $V$. A map $f: H \rightarrow V$ is a 1-cocycle over $\pi$ if for any $h_{1}, h_{2} \in H, f\left(h_{1} h_{2}\right)=f\left(h_{1}\right)+\pi\left(h_{1}\right) f\left(h_{2}\right)$; a map $f: H \rightarrow V$ is a 
1-coboundary over $\pi$ if there exists $v \in V$ such that $f(h)=v-\pi(h) v$ for all $h \in H$; 1-cohomology group $H^{1}(H, \pi)$ over $\pi$ is the quotient of the group $Z^{1}(H, \pi)$ of all 1-cocycles over $\pi$ by the group $B^{1}(H, \pi)$ of all 1-coboundaries over $\pi$. (We remark that it is clear that $Z^{1}(H, \pi)$ and $B^{1}(H, \pi)$ are abelian groups with the usual pointwise addition, and $B^{1}(H, \pi) \subset Z^{1}(H, \pi)$, therefore $H^{1}(H, \pi)$ makes sense.)

2. (D2) (Kazhdan's property T). A locally compact group $H$ is Kazhdan if for all unitary (orthogonal) representations $\pi$ of $H$ on complex (real) Hilbert spaces, $H^{1}(H, \pi)=0$. (We remark this is an equivalent definition among variety of other equivalent definitions of Kazhdan's property $\mathrm{T}$, see [2].)

3. (D3) (Strong vanishing condition SVC) A group $H$ satisfies SVC if for all finite dimensional representations $\pi, H^{1}(H, \pi)=0$. (We remark that SVC first appeared in [4]. Irreducible lattices in connected, semisimple Lie group without compact factors, with $\mathbb{R}$-rank at least 2 satisfy SVC, as well as lattices in connected, semisimple Lie group without compact factors, each simple factor has $\mathbb{R}$-rank at least 2 . They are also Kazhdan groups. See [14]).

(D4-8) define the infinitesimal rigidity.

1. (D4) $\alpha: \Gamma \rightarrow \mathcal{X}^{r}(E)$ a $C^{r}$-cocycle of $\tilde{\rho}$ with coefficients in vector bundle $E$ if

$$
\alpha\left(\gamma_{1} \gamma_{2}\right)=\alpha\left(\gamma_{1}\right)+\left(\tilde{\rho}\left(\gamma_{1}\right)\right)_{*} \alpha\left(\gamma_{2}\right) .
$$

2. (D5) $\alpha: \Gamma \rightarrow \mathcal{X}^{r}(E)$ is a $C^{s}$-coboundary ( $L^{2}$-coboundary) of $\tilde{\rho}$ with coefficients in vector bundle $E$ if there exists $v \in \mathcal{X}^{s}(E)\left(\mathcal{X}^{L^{2}}(E)\right)$ such that

$$
\alpha(\gamma)=v-(\tilde{\rho}(\gamma))_{*} v
$$

We also say that $v$ is a $C^{s}$ - (or an $L^{2}$-) coboundary of $\alpha$.

3. (D6) The quotient group $H_{r, s}^{1}(\tilde{\rho}, E)\left(H_{r, L^{2}}^{1}(\tilde{\rho}, E)\right)$ of the additive group of all $C^{r}$-cocycles by the additive group of all $C^{s}$-coboundaries $\left(L^{2}\right.$ coboundaries) with coefficients in vector bundle $E$ is the first cohomology group of $\tilde{\rho}$ with coefficients $E$.

4. (D7) $\tilde{\rho}$ is $(r, s)$-infinitesimal $\left(\left(r, L^{2}\right)\right.$-infinitesimal) rigid if $H_{r, s}^{1}(\tilde{\rho}, E)=$ $0\left(H_{r, L^{2}}^{1}(\tilde{\rho}, E)=0\right)$. 
5. (D8) Let $\tilde{\rho}$ be the tangent action induced from $\rho$. $\rho$ is $(r, s)$-infinitesimal $\left(\left(r, L^{2}\right)\right.$-infinitesimal $)$ rigid if $H_{r, s}^{1}(\tilde{\rho}, T M)=0$ $\left(H_{r, L^{2}}^{1}(\tilde{\rho}, T M)=0\right) ; \rho$ is $C^{0}$-infinitesimal rigid if $H_{0,0}^{1}(\tilde{\rho}, T M)=0$, $\rho$ is $C^{\infty}$-infinitesimal rigid if $H_{\infty, \infty}^{1}(\tilde{\rho}, T M)=0$.

(D9-10) essentially define notions at the tangential level.

1. (D9) (flat actions) Let $\tilde{\rho}$ be an action of a discrete group $\Gamma$ on a flat vector bundle $E=M \times \mathbb{R}^{n_{0}}$ covering an action $\rho$ of $\Gamma$ on $M$. $\tilde{\rho}$ is said to be flat associated with a homomorphism $\pi: \Gamma \rightarrow G L\left(n_{0}, \mathbb{R}\right)$ if there exists a $C^{\infty}$-framing $\sigma$ (i.e., a set of $C^{\infty}$ sections $\left\{X_{1}, \ldots, X_{n}\right\}$ of $E \rightarrow$ $M$, called linearizing framing) such that $\tilde{\rho}(\gamma)_{x}(\sigma(x))=\sigma(\rho(\gamma)(x)) \pi(\gamma)$ for all $\gamma \in \Gamma$. $\rho$ is said to be tangentially flat associated with a homomorphism $\pi: \Gamma \rightarrow G L(n, \mathbb{R})$ if $T M$ is flat and $\tilde{\rho}$ is the action on tangent bundle which is flat associated with $\pi$. (We note that one common feature of type 2 and type 3 standard actions is the tangential flatness).

2. (D10) A homomorphism $\pi: \Gamma \rightarrow G L(n, \mathbb{R})$ has compact part if there exists an invariant vector subspace $V \subset \mathbb{R}^{n}$, such that the spectrum of the restriction of $\pi(\gamma)$ to $V \operatorname{Spect}\left(\left.\pi(\gamma)\right|_{V}\right) \subset S^{1}$ for all $\gamma \in \Gamma$, where $S^{1} \subset \mathbb{C}$ is the set of complex numbers of absolute value 1 .

\subsection{Abelian subgroups of lattices.}

Abelian group actions contained in a lattice group action impose many constraints for possible perturbations, they contribute greatly to the rigidity of the action $[6,7]$. We collect some facts concerning the existence and the structure of abelian subgroups of a lattice group $\Gamma$ in a connected semisimple Lie group $G \subset G L\left(n_{0}, \mathbb{R}\right)$ for some integer $n_{0}>0$. The following is taken from [17]. We assume that $G$ is self-adjoint; i.e, $G$ is stable under the inverse transpose $\Theta, \Theta(G)=G$. The automorphism $\Theta$ of $G$ induces an automorphism $\theta$ of the Lie algebra $\mathfrak{g}$ of $G$. Take a Cartan subalgebra $\mathfrak{h}$ of $\mathfrak{g}$; i.e., a subalgebra of $\mathfrak{g}$ that is maximal among abelian $\theta$-stable subalgebras. The Cartan subgroup $H$ of $G$ corresponding to $\mathfrak{h}$ is the centralizer in $G$ of $\mathfrak{h}$; i.e., the set of all $g \in G$ such that $\operatorname{Ad}(g) X=X$ for all $X \in \mathfrak{h}$. If $\mathfrak{g}=\mathfrak{g}_{1} \oplus \cdots \oplus \mathfrak{g}_{h}$ is the decomposition of $\mathfrak{g}$ into simple ideas, then a Cartan subalgebra $\mathfrak{h}$ intersects each of the ideas $\mathfrak{g}_{\mathfrak{i}}$. A Cartan subgroup is abelian and diagonalizable over $\mathbb{C}$. If $G$ is noncompct, different Cartan subgroups 
may not be conjugate but there are only finitely many nonconjugate $\Theta$ stable Cartan subgroups. Following theorem asserts the existence of certain abelian subgroups in $G$.

Theorem 2.1. (Theorem of [15] p. 211, Theorem 2.8 of [17]) Let $G$ be a connected semisimple Lie group and $\Gamma$ be a lattice in $G$. Let $H$ be a Cartan subgroup, then $\Gamma$ intersects a conjugate of $H$ in a uniform lattice.

Fix a standard abelian group action of $H$ on $n$-dimensional compact manifold $M$ preserving the Lebesgue measure. We call a non-trivial homomorphism $\lambda: H \rightarrow \mathbb{R}(\mathbb{R}$ as an additive group) a Lyapunov exponent for the tangent bundle $T M$ if for all $h \in H$, almost all $x \in M$, there exists $0 \neq u \in T_{x} M$ so that the exponential growth rate $\lim _{n \rightarrow \infty} \frac{1}{n} \log \left\|D \rho\left(h^{n}\right)(u)\right\|$ for $h$ is given by $\lambda(h)$ (independent of $x$ ). For a Lyapunov exponent $\lambda$, let $E_{\lambda}(x) \subset T_{x} M$ be the set of all $u$ as above (union $\{0\}$ ). Since the action is standard, $E_{\lambda}(x)$ is constant distribution for each Lyapunov exponent $\lambda$ and of class $C^{\infty}$. Moreover, the tangent bundle has a natural $H$-invariant splitting: $T M=\sum E_{\lambda}$.

For a flat action of abelian group $H$ on a flat bundle $E \rightarrow M$, we may define the notion of Lyapunov exponents and Lyapunov subspaces and we may also define the notions for homomorphism $H \rightarrow G L(n, \mathbb{R})$ in an obvious way. In case of the flat bundle action or tangentially flat action we shall abuse the notation to use the notions for any three of the situations if there is no confusion. Later on we shall always assume that our bundles and actions are flat.

We use $\mathcal{A}$ to denote a finitely generated abelian group. Let the action of $\mathcal{A}$ be flat on flat bundle $E \rightarrow M$. We call a Lyapunov exponent $\lambda$ real if for any $a \in \mathcal{A}, u \in E_{\lambda}, \pi(a) u=\exp (\lambda(a)) u$ (in other words, the Lyapunov space $E_{\lambda}$ corresponding to $\lambda$ is also the common eigenspace for $\mathcal{A}$ corresponding to $\exp (\lambda)$ ); we call a real Lyapunov exponent $\lambda$ almost multiplicity free if $\mu$ is another Lyapunov exponent with $\mu=c \lambda$, then $\mu$ is real and one of $c, 1 / c$ is an integer. We call a Lyapunov exponent $\lambda$ multiplicity free if $c=1$ ( $c$ as above).

We say that a completely reducible representation $\pi: \Gamma \rightarrow G L(n, \mathbb{R})$ is of (almost) Lyapunov multiplicity free type, if for each $\pi$-invariant non-trivial subspace $V \subset \mathbb{R}^{n}$, there exist

1. A finitely generated abelian group $\mathcal{A} \subset \Gamma$;

2. A real Lyapunov exponent $\lambda: \mathcal{A} \rightarrow \mathbb{R}$ with Lyapunov space $E_{\lambda}$ so that $E_{\lambda} \cap V \neq\{0\}$ and $\lambda$ is (almost) multiplicity free; 
3. There exists $a \in \mathcal{A}$, such that $\operatorname{dim} E_{0}(a)=\min _{\gamma \in \Gamma} \operatorname{dim} E_{0}(\gamma)$, where for $\gamma \in \Gamma$ denote by $E_{0}(\gamma)$ the Lyapunov space of $\pi(\gamma)$ corresponding to Lyapunov exponent 0 . Moreover, if $\operatorname{dim} E_{0}(a)>0$, all unit eigenvalues of $\pi(a)$ are 1.

4. Let $\mu$ be another Lyapunov exponent not proportional to $\lambda$ ( $\lambda$ as in (2)). Then $a$ in (3) may be chosen so that $\lambda(a)<0$ and $\mu(a)>0$.

The following result gives a criterion of Lyapunov multiplicity freeness.

Proposition 2.2. Let $G$ be a connected semisimple linear Lie group, $\pi_{0} a$ restriction to $\Gamma$ of a homomorphism of $\pi: G \rightarrow G L(n, \mathbb{R})$.

1. If $\pi$ is hyperbolic (i.e., there exists $\gamma \in G$ such that the eigenvalues of $\pi(\gamma)$ have modulus different from 1. Such $\pi(\gamma)$ is said to be hyperbolic), then $\pi_{0}$ is hyperbolic. In other words, there exists $\gamma_{1} \in \Gamma$ such that $\pi_{0}\left(\gamma_{1}\right)$ is hyperbolic.

2. Let $m=\max _{\gamma \in G}\{l \in \mathbb{Z}: \pi(\gamma)$ has $l$ eigenvalues of modulus different from 1$\}$. Then there exists $\gamma_{1} \in \Gamma$ such that $\pi_{0}\left(\gamma_{1}\right)$ has $m$ eigenvalues of modulus different from 1.

3. If $G$ is an $\mathbb{R}$-split linear group and for any irreducible constituent $\pi^{\prime}$ of $\pi$, there exists a multiplicity free weight $\lambda^{\prime} \neq 0$ (i.e., any weight of $\pi$ is not proportional to $\left.\lambda^{\prime}\right)$. Then $\pi_{0}$ is of Lyapunov multiplicity free type.

Proof. It is well-known that any connected linear semisimple group $G$ can be realized as the connected component of identity in $\mathbf{G}_{\mathbb{R}}$ of a connected, selfjoint, semi-simple algebraic group $\mathbf{G} \subset G L(n, \mathbb{C})$ defined over $\mathbb{R}$. Without loss of generality, we assume that $G$ is such a realization. Let $\operatorname{pol}(\gamma)$ be the polar part of $\gamma_{s}$, where $\gamma_{s}$ is the semisimple part of the Jordan decomposition $\gamma=\gamma_{s} \gamma_{u}$ of $\gamma$. It is well-known that $\operatorname{pol}(\gamma) \in G$, and it is contained in a maximum $\mathbb{R}$-split abelian group $S$ which is contained in a Cartan subgroup $H$. (We refer the reader to $\S 1$, p. 297 of [17] and the references there for the above discussion.)

(1) Since $\pi(\gamma)$ is hyperbolic, $\pi(\operatorname{pol}(\gamma))$ is hyperbolic. Denote by $\mathcal{A}$ the intersection of $\Gamma$ with a conjugate of $H$ (without loss of generality we assume that the conjugate is $H$ itself) so that $\mathcal{A}$ is a uniform lattice in $H$. Let $\left\{\lambda_{1}, \ldots, \lambda_{s}\right\}$ be the set of all Lyapunov exponents of $\mathcal{A}$ corresponding to $\pi_{0}$. Then they extend to Lyapunov exponents of $H$ corresponding to $\pi$. The 
Lyapunov exponents for matrix are the logarithms of the absolute values of the eigenvalues, therefore, for all $i=1, \ldots, s \lambda_{i}(\operatorname{pol}(\gamma)) \neq 0$.

As is well-known for Cartan subgroups of semisimple connected Lie groups, $H=T \times B$ (direct) where $T$ is the maximum compact subgroup of $H$ and $B$ is a vector subgroup such that $\operatorname{Ad} B$ is diagonalizable over the reals. It is clear that $\left.\lambda_{i}\right|_{T}=0$. Let $K_{i}$ be the kernel of $\lambda_{i}$, then $K_{i}=T \times B_{i}$ for some codimension 1 hyperplane $B_{i}$ in $B$. Since $H-\cup_{i=1}^{s} K_{i}=T \times \cup_{i=1}^{s}\left(B-B_{i}\right)$ and each connected component $\cup_{i=1}^{s}\left(B-B_{i}\right)$ is a non-empty open cone in $B,\left(H-\cup_{i=1}^{s} K_{i}\right) \cap \mathcal{A}$ is non-empty. Let $\gamma_{1}$ be in the intersection. Then the eigenvalues of $\pi\left(\gamma_{1}\right)$ are non-zero, hence $\pi\left(\gamma_{1}\right)$ and therefore $\pi_{0}$ are hyperbolic.

(2) Same argument as in the proof of (1). We replace all Lyapunov exponents $\left\{\lambda_{1}, \ldots, \lambda_{s}\right\}$ by non-trivial Lyapunov exponents.

(3) Let $H$ in the proofs of (1), (2) be such that contains an $\mathbb{R}$-split abelian group $A$. Let $\mathfrak{a}$ be the Lie algebra of $A$. Then $\mathfrak{a}$ is a Cartan subalgebra. Homomorphism $\pi: G \rightarrow G L(n, \mathbb{R})$ induces a homomorphism of the Lie algebras of $G$ and $G L(n, \mathbb{R})$. The weights of $\pi$ (with respect to $\mathfrak{a}$ ) correspond to the Lyapunov exponents of $\pi(H)$. Since $H$ is $\mathbb{R}$-split, all the Lyapunov spaces of $\pi(H)$ are eigenspaces. Hence all Lyapunov exponents are real.

To verify condition (4) in the definition of Lyapunov multiplicity freeness, we notice that $\gamma_{1}$ in the proofs of (1) and (2) may be taken to be in $T \times$ $(B-U)$, where $U$ is the sum of any given finitely many hyperspaces. So we may assume that $\lambda(a)$ and $\mu(a)$ are non-zero. Moreover, we may assume that $\lambda(a)<0$ and $\mu(a)>0$ by Lemma 4.1 proved later.

By definition, $\pi_{0}$ is of Lyapunov multiplicity free type.

\subsection{The Livsic theorem.}

We list in Theorems 2.3, 2.4 some well-known results about the cohomological equation for diffeomorphisms. We thank the referee for pointing out a result of Livsic (Theorem 2.3 (3) below) that replaces a weeker version in our earlier writing.

Theorem 2.3. (the Livsic theorem) Let $T: M \rightarrow M$ be an Anosov diffeomorphism with a dense orbit.

1. Let $f$ be a Hölder continuous function on $M$. Then the equation

$$
f(x)=\phi(x)-\phi(T x)
$$


has a Hölder continuous solution $\phi$ iff for any periodic point $p \in M$ with period $m$, the closing condition $\sum_{i=1}^{m} f\left(T^{i} p\right)=0$ is satisfied.

2. Let $f$ be a $C^{\infty}$ function on $M$. Then $\phi$ in (1) is $C^{\infty}$.

3. Let $T: M \rightarrow M$ be an Anosov $C^{2}$-diffeomorphism preserving a Lebesgue measure, let $f$ be a Hölder continuous function. If (2.1) has a measurable solution $\phi$, then $\phi$ coincides almost everywhere with a continuous function $\phi^{\prime}$ which is also a solution of (2.1). In particular, the closing conditions for all periodic points are satisfied.

(1) appeared in [10] (Theorem 1.1). (2) was proved in [12]. (3) appeared in [11] ( $\S 6$, Theorem 9).

The following proposition is a Livsic type theorem for certain partially hyperbolic diffeomorphisms.

Proposition 2.4. Let $M$ be as before and $N$ be a connected smooth manifold, $f: M \times N \rightarrow \mathbb{R}$ be a $C^{\infty}$ function. Define $T: M \times N$ by $(m, n) \rightarrow(T m, n)$. For each $n \in N$ denote by $f_{n}: M \rightarrow M$ the restriction of $f$ to the $T$-invariant compact manifold $M \times\{n\}$. Assume that $T_{0}: M \rightarrow M$ is a $C^{\infty}$-transitive Anosov diffeomorphism satisfying the closing condition at each periodic point $\left(\right.$ for $\left.f_{n}\right)$. Then

1. for any function $\eta: N \rightarrow \mathbb{R}$, there exists a unique function $\phi: M \times$ $N \rightarrow \mathbb{R}$ such that $f(m, n)=\phi(m, n)-\phi(T m, n), \phi(p, n)=\eta(n)$ and $\phi(m, n)$ is of class $C^{\infty}$ for any fixed $n \in N$;

2. if $\eta$ in (1) is of class $C^{\infty}$, then $\phi$ is of class $C^{\infty}$.

Proof. (1) is a straightforward corollary of Theorem 2.3. (2) is a reformulation of Theorem 2.2 of [12].

\subsection{Some algebraic lemmas and regularity results.}

The following results are simple but important for the rest of the paper. It illustrates the following simple phenomena: An $\mathbb{R}^{n}$-valued function $f$ on $M$ is regular if the projections of $f$ to sufficiently many subspaces are regular.

Lemma 2.5. Let $\pi: \Gamma \rightarrow G L(n, \mathbb{R})$ be a homomorphism. Assume that there exist $d$ elements $\beta_{1}, \ldots, \beta_{d} \in \Gamma$ and $d$ non-trivial vector subspaces 
$V_{1}, \ldots, V_{d} \subset \mathbb{R}^{n}$ such that each $V_{i}$ is the sum of a subset of generalized eigenspaces of $\pi\left(\beta_{i}\right)$, and for every $\pi$-invariant subspace $V \subset \mathbb{R}^{n}$ there exists at least one $1 \leq i \leq d$ such that $V_{i} \cap V \neq\{0\}$. Then there exist an integer $s \geq 1$ and

- $s$ elements $\gamma_{1}, \ldots, \gamma_{s} \in \Gamma$, for each $\gamma_{j}$ there exist $1 \leq i_{j} \leq d$ and $\delta_{i_{j}} \in \Gamma$ such that $\gamma_{j}=\delta_{i_{j}} \beta_{i_{j}} \delta_{i_{j}}^{-1}$;

- $s$ non-trivial vector subspaces $E_{1}, \ldots, E_{s} \subset \mathbb{R}^{n}$, for each $E_{j}(1 \leq j \leq s)$ $E_{j}=\pi\left(\delta_{i_{j}}\right) V_{j_{i}}$

- $s$ vector subspaces $F_{1}, \ldots, F_{s} \subset \mathbb{R}^{n}$

such that

1. for each $1 \leq i \leq s, E_{i}, F_{i}$ are $\pi\left(\gamma_{i}\right)$-invariant, $\operatorname{dim}\left(E_{i}\right)+\operatorname{dim}\left(F_{i}\right)=n$, $E_{i}+F_{i}=\mathbb{R}^{n}$, and both $E_{i}$ and $F_{i}$ are generalized eigenspaces of $\gamma_{i}$ if $1 \leq \operatorname{dim}\left(E_{i}\right), \operatorname{dim}\left(F_{i}\right) \leq(n-1)$;

2. $\cap_{i=1}^{s} F_{i}=\{0\}$.

Proof. Let the $V_{1}$ be the sum of a subset of generalized eigenspaces of $\pi\left(\beta_{1}\right)$, and $V_{1}^{\perp}$ be the sum of complementary generalized eigenspaces of $\pi\left(\beta_{1}\right)$. Consider elements $\gamma \beta_{1} \gamma^{-1} . \pi(\gamma) V_{1}, \pi(\gamma) V_{1}^{\perp}$ are vector subspaces invariant under $\pi\left(\gamma \beta_{1} \gamma^{-1}\right)$; they are both the sum of generalized eigenspaces of $\pi\left(\gamma \beta_{1} \gamma^{-1}\right)$. Consider $W=\cap_{\gamma \in \Gamma} \pi(\gamma) V_{1}^{\perp} ; W$ is $\pi$-invariant and clearly there exist finite number of elements $\delta_{1}, \ldots, \delta_{s_{1}} \in \Gamma$ such that $W=\cap_{i=1}^{s_{1}} \pi\left(\delta_{i}\right) V_{1}^{\perp}$. We denote $\gamma_{i}=\delta_{i} \gamma_{0} \delta_{i}^{-1}, E_{i}=\pi\left(\delta_{i}\right) V_{1}, F_{i}=\pi\left(\delta_{i}\right) V_{1}^{\perp}$ for $i=1, \ldots, s_{1}$.

Since $W$ is $\pi$-invariant and $W \cap V_{1}=0$, it intersects $V_{i}$ for some $i=$ $2, \ldots, d$. By induction, our assertion is obvious.

We have a special version of the lemma for homomorphism without compact part.

Lemma 2.6. Let $\pi: \Gamma \rightarrow G L(n, \mathbb{R})$ be a homomorphism with no compact part. Then there exist an integer $s \geq 1$ and

- $s$ elements $\gamma_{1}, \ldots, \gamma_{s} \in \Gamma$;

- $s$ non-trivial vector subspaces $E_{1}, \ldots, E_{s} \subset \mathbb{R}^{n}$;

- $s$ vector subspaces $F_{1}, \ldots, F_{s} \subset \mathbb{R}^{n}$

such that

1. for each $1 \leq i \leq s, E_{i}, F_{i}$ are $\pi\left(\gamma_{i}\right)$-invariant, $\operatorname{dim}\left(E_{i}\right)+\operatorname{dim}\left(F_{i}\right)=n$, $E_{i}+F_{i}=\mathbb{R}^{n}, E_{i}$ is the sum of generalized eigenspaces of $\gamma_{i}$ corresponding to eigenvalues of absolute values less than $1, F_{i}$ is $\{0\}$ or the sum of generalized eigenspaces of $\gamma_{i}$ corresponding to other eigenvalues; 
2. $\cap_{i=1}^{s} F_{i}=\{0\}$.

Lemma 2.7. Assume the notation in Lemma 2.5. Let $P_{i}: \mathbb{R}^{n} \rightarrow E_{i}$ be the projection operator associated with the decomposition $\mathbb{R}^{n}=E_{i} \oplus F_{i}$. Then the homomorphism $h: \mathbb{R}^{n} \rightarrow\left(\mathbb{R}^{n}\right)^{s}, v \in \mathbb{R}^{n} \mapsto\left(P_{1} v, \ldots, P_{s} v\right)$ is injective.

Proof. Let $v \in \mathbb{R}^{n}$ such that $h(v)=0$. Then for all $i=1, \ldots, s P_{i} v=0$. Since $P_{i} v=0$ iff $v \in F_{i}$, we have $v \in \cap_{i=1}^{s} F_{i}=\{0\}$. So $v=0$.

Corollary 2.8. Assume the notation in Lemmas 2.5, 2.7. Let $S$ be a dense set in $M, f: S \subset M \rightarrow \mathbb{R}^{n}$ be a map such that $P_{i} \circ f$ extends to a $C^{r}$ map for all $1 \leq i \leq s$. Then $f$ extends to a $C^{r} \operatorname{map} M \rightarrow \mathbb{R}^{n}$.

Proof. Let $U=h\left(\mathbb{R}^{n}\right)$. Then $h: \mathbb{R}^{n} \rightarrow U$ is an isomorphism. Denote $h^{-1}$ : $U \rightarrow \mathbb{R}^{n}$ the inverse of $h: \mathbb{R}^{n} \rightarrow U$. Obviously, $h^{-1}$ is of class $C^{\infty}$. For each $i$ let $f_{i}: M \rightarrow \mathbb{R}^{n}$ be the $C^{r}$ map so that the restriction to $\left.S f_{i}\right|_{S}=P_{i} \circ f$. For any $x \in X$, let $x_{k} \in S$ so that $x_{k} \rightarrow x$ as $k \rightarrow \infty$, then the points in $U\left(P_{1} \circ f\left(x_{k}\right), \ldots, P_{s} \circ f\left(x_{k}\right)\right)=\left(f_{1}\left(x_{k}\right), \ldots, f_{s}\left(x_{k}\right)\right) \rightarrow\left(f_{1}(x), \ldots, f_{s}(x)\right)$. Hence $\left(f_{1}(x), \ldots, f_{s}(x)\right) \in U$ also ( $U$ is a closed vector subspace). We thus obtain a $C^{r}$ function $h^{-1}\left(f_{1}(x), \ldots, f_{s}(x)\right)$ from $M \rightarrow \mathbb{R}^{n}$ that coincides $f$ at every point in the dense set $S$. In other words, $f$ extends to a $C^{r}$ map.

Same argument proves the following.

Proposition 2.9. Assume the notation in Lemmas 2.5-2.8. Then $f: M \rightarrow$ $\mathbb{R}^{n}$ is of class $C^{r}$ iff $P_{i} \circ f$ is of class $C^{r}$ for all $1 \leq i \leq s$.

For the convenience of later use, we give a criterion of the regularity of an $\mathbb{R}^{n}$-valued function $f$. Let $\pi: \Gamma \rightarrow G L(n, \mathbb{R})$ be a homomorphism. For $\gamma \in \Gamma$, we use $E(\gamma), F(\gamma)$ to denote the sum of some subsets of generalized eigenspaces for $\pi(\gamma)$, so that $\mathbb{R}^{n}=E(\gamma) \oplus F(\gamma)$ is a direct sum. We denote by $f_{(E(\gamma), F(\gamma))}$ the projection of $f$ to the first factor $E(\gamma)$ with respect to this decomposition. For any $\gamma^{\prime} \in \Gamma$, we let $E\left(\gamma^{\prime} \gamma \gamma^{\prime-1}\right)=\pi\left(\gamma^{\prime}\right) E(\gamma), F\left(\gamma^{\prime} \gamma \gamma^{\prime-1}\right)=\pi\left(\gamma^{\prime}\right) F(\gamma)$. It is easy to see that $\pi\left(\gamma^{\prime}\right) E(\gamma), \pi\left(\gamma^{\prime}\right) F(\gamma)$ are the sum of some subsets of generalized eigenspaces for $\pi\left(\gamma^{\prime} \gamma \gamma^{\prime-1}\right)$, and $\mathbb{R}^{n}=\pi\left(\gamma^{\prime}\right) E(\gamma) \oplus \pi\left(\gamma^{\prime}\right) F(\gamma)$ is a direct sum.

Theorem 2.10. Let $f: M \rightarrow \mathbb{R}^{n}$ be an $\mathbb{R}^{n}$-valued function, $\pi: \Gamma \rightarrow$ $G L(n, \mathbb{R})$ a homomorphism. Assume that 
1. for each $\pi$-invariant space $V \subset \mathbb{R}^{n}$, there exists $\gamma \in \Gamma$, and a decomposition $\mathbb{R}^{n}=E(\gamma) \oplus F(\gamma)$ into a direct sum of the sum of some subsets of generalized eigenspaces of $\pi(\gamma)$, such that $E(\gamma) \cap V \neq 0$ and $f_{(E(\gamma), F(\gamma))}$ is of class $C^{r}$;

2. for each $\gamma^{\prime} \in \Gamma$ and $\gamma$ as in (1), $f_{\left(E\left(\gamma^{\prime} \gamma \gamma^{\prime-1}\right), F\left(\gamma^{\prime} \gamma \gamma^{\prime-1}\right)\right)}$ is also of class $C^{r}$.

Then $f$ is of class $C^{r}$.

Proof. In view of the proof of Lemma 2.7, it is enough to prove the following: There exist finitely many elements $\beta_{1}, \ldots, \beta_{N} \in \Gamma$, such that (i) for each $\beta_{i}$, there is a decomposition $\mathbb{R}^{n}=E\left(\beta_{i}\right) \oplus F\left(\beta_{i}\right)$ into a direct sum of the sum of some subsets of generalized eigenspaces of $\pi\left(\beta_{i}\right)$ such that $f_{\left(E\left(\beta_{i}\right), F\left(\beta_{i}\right)\right)}$ is of class $C^{r}$; (ii) $\cap_{i=1}^{N} F\left(\beta_{i}\right)=\{0\}$.

From the assumption (1), we choose $\gamma_{1} \in \Gamma$ such that $\mathbb{R}^{n}=E\left(\gamma_{1}\right) \oplus F\left(\gamma_{1}\right)$ is a direct sum of the sum of some subsets of generalized eigenspaces of $\pi\left(\gamma_{1}\right)$, and $f_{\left(E\left(\gamma_{1}\right), F\left(\gamma_{1}\right)\right)}$ is of class $C^{r}$. We let $W_{1}=\cap_{\gamma^{\prime} \in \Gamma} F\left(\gamma^{\prime} \gamma_{1} \gamma^{\prime-1}\right)=$ $\cap_{\gamma^{\prime} \in \Gamma} \pi\left(\gamma^{\prime}\right) F\left(\gamma_{1}\right)$, which is $\pi$-invariant. We may choose finitely many elements $\delta_{1}, \ldots, \delta_{d_{1}} \in \Gamma$ such that $W_{1}=\cap_{i=1}^{d_{1}} \pi\left(\delta_{i}\right) F\left(\gamma_{1}\right)$. We let $\beta_{i}=\delta_{i} \gamma_{1} \delta_{i}^{-1}$ for $i=1, \ldots, d_{1}$. By the assumption (2), $f_{\left(E\left(\beta_{i}\right), F\left(\beta_{i}\right)\right)}$ is of class $C^{r}$ for $i=1, \ldots, d_{1}$.

Since $W_{1}$ is $\pi$-invariant and the assumption (1), we choose $\gamma_{2} \in \Gamma$ such that $\mathbb{R}^{n}=E\left(\gamma_{2}\right) \oplus F\left(\gamma_{2}\right)$ is a direct sum of the sum of some subsets of generalized eigenspaces of $\pi\left(\gamma_{2}\right)$ and $f_{\left(E\left(\gamma_{2}\right), F\left(\gamma_{2}\right)\right)}$ is of class $C^{r}$. We let $W_{2}^{\prime}=$ $\cap_{\gamma^{\prime} \in \Gamma} F\left(\gamma^{\prime} \gamma_{2} \gamma^{-1}\right)=\cap_{\gamma^{\prime} \in \Gamma} \pi\left(\gamma^{\prime}\right) F\left(\gamma_{2}\right)$, which is $\pi$-invariant. We may choose finitely many elements $\delta_{d_{1}+1}, \ldots, \delta_{d_{2}} \in \Gamma$ such that $W_{2}^{\prime}=\cap_{i=d_{1}+1}^{d_{2}} \pi\left(\delta_{i}\right) F\left(\gamma_{2}\right)$. We let $\beta_{i}=\delta_{i} \gamma_{2} \delta_{i}^{-1}$ for $i=d_{1}+1, \ldots, d_{2}$. We have $W_{2}:=\cap_{i=1}^{d_{2}} F\left(\beta_{i}\right)=$ $W_{1} \cap W_{2}^{\prime}$, and $W_{2}$ is a proper subspace of $W_{1}$. By the assumption (2), $f_{\left(E\left(\beta_{i}\right), F\left(\beta_{i}\right)\right)}$ is of class $C^{r}$ for $i=d_{1}+1, \ldots, d_{2}$.

Since $W_{2}$ is $\pi$-invariant and the assumption (1), we may repeat the process to obtain $\beta_{d_{2}+1}, \ldots, \beta_{d_{3}} \in \Gamma$ such that $f_{\left(E\left(\beta_{i}\right), F\left(\beta_{i}\right)\right)}$ is of class $C^{r}$ for $i=d_{2}+1, \ldots, d_{3}$, and $W_{3}:=\cap_{i=1}^{d_{3}} F\left(\beta_{i}\right)$ is a proper subspace of $W_{2}$. We keep doing the process, obtain a proper inclusion of subspaces $W_{k+1} \subset W_{k}$. It is obvious that after a finitely many steps, $W_{j}=0$.

For such $\beta_{1}, \ldots, \beta_{N} \in \Gamma$, we have that for each $\beta_{i}$, there is a decomposition $\mathbb{R}^{n}=E\left(\beta_{i}\right) \oplus F\left(\beta_{i}\right)$ into a direct sum of the sum of some subsets of generalized eigenspaces of $\pi\left(\beta_{i}\right)$ such that $f_{\left(E\left(\beta_{i}\right), F\left(\beta_{i}\right)\right)}$ is of class $C^{r}$, and $\cap_{i=1}^{N} F\left(\beta_{i}\right)=\{0\}$. 


\section{3. $(r, 0)$-infinitesimal rigidity.}

In this section we establish $\left(r, L^{2}\right)$ - and $(r, 0)$-infinitesimal rigidity for actions of Kazhdan, SVC group $\Gamma$ with dense periodic points $\Omega$. We remark that the type 3 standard actions, or the actions on tori $\mathbb{T}^{n}$ via a homomorphism $\Gamma \rightarrow S L(n, \mathbb{Z})$ or on nilmanifolds $M$ via a homomorphism $\Gamma \rightarrow \operatorname{Aut}(M)$ are primary examples of actions with dense periodic points. One isolated type 2 standard action example can also be constructed as follows: if $\Lambda$ is a torsion free, cocompact, arithmetic subgroup in a connected semisimple Lie group with trivial center and no compact factors, the action of $\Lambda$ on $G / \Lambda$ by left translation has dense periodic points (a corollary of 6.2.4 of $[19])$.

\subsection{Actions with dense periodic points: $\pi$ has no compact part.}

In this subsection, we will show that a tangentially flat action $\rho$ associated with a homomorphism $\pi: \Gamma \rightarrow G L(n, \mathbb{R})$ is $(r, 0)$-infinitesimal rigid provided that $\pi$ has no compact part and $\rho$ has dense periodic points $\Omega(\rho)$.

The following is essentially a lemma of Hurder [5].

Lemma 3.1. Let $\tilde{\rho}$ be an action on a vector bundle $E \rightarrow M$ (by vector bundle automorphisms) covering $\rho$. Let $\Omega=\Omega(\rho)$ be the set of periodic points of $\rho$. Assume that $\Gamma$ satisfies SVC. Then for every $C^{r}$-cocycle $\alpha$ with coefficients in $E$ there exists a set function $v_{0}: \Omega \rightarrow T_{\Omega} M$, such that $\left.\alpha(\gamma)\right|_{\Omega}=\left.v_{0}\right|_{\Omega}-\left.(\tilde{\rho}(\gamma))_{*} v_{0}\right|_{\Omega}$.

Proof. Take $p \in \Omega$, let $O(p)=\rho(\Gamma) p=\left\{p_{1}=p, \ldots, p_{N}\right\}$. $\tilde{\rho}$ induces an action on the sum $E_{O(p)}$ of fibers over $\left\{p_{1}, \ldots, p_{N}\right\}$; or equivalently, it induces a representation $\pi_{p}$ of $\Gamma$ on vector space $W=\oplus_{i=1}^{N} E_{p_{i}}$ where $E_{p_{i}}$ is the fiber of $E$ over $p_{i}$. Since $\left.\alpha\right|_{O(p)}$ can be viewed as a cocycle of $\Gamma$ with coefficients in $\pi_{p}$, there exists $v_{0}: O(p) \rightarrow E_{O(p)}$ such that for every $\gamma \in \Gamma$, $\left.\alpha(\gamma)\right|_{O(p)}=\left.v_{0}\right|_{O(p)}-\left.(\tilde{\rho}(\gamma))_{*} v_{0}\right|_{O(p)}$ by SVC. Do this for all orbits of points in $\Omega$, our lemma follows.

Proposition 3.2. Let $E=M \times \mathbb{R}^{n_{0}}$ be a trivial bundle, $\tilde{\rho}$ be a flat action of $\Gamma$ on $E$ associated with a homomorphism $\pi: \Gamma \rightarrow \mathbb{R}^{n_{0}}$ covering an action $\rho$ of $\Gamma$ on $M$. Assume that $\Gamma$ is SVC group, $\pi$ has no compact part and the set $\Omega(\rho)$ of periodic points of $\rho$ is dense in $M$. Then $v_{0}$ (as in Lemma 3.1) extends to a continuous coboundary for $C^{r}$-cocycle $\alpha$. 
Proof. Without loss of generality we assume that the trivialize $E=M \times \mathbb{R}^{n_{0}}$ is given by the linearizing framing with respect to which $\tilde{\rho}(\gamma)$ is given by the homomorphism $\pi$. For each $1 \leq i \leq s$, let $E_{i}, F_{i}$ be as in Lemma 2.6', let the subbundle $\bar{E}_{i}$ correspond to $E_{i}$ in an obvious way. It is easy to see that $\left.\operatorname{Id}\right|_{\bar{E}_{i}}-\left(\tilde{\rho}\left(\gamma_{i}\right)\right)_{*}$ is an invertible operator on the set $\mathcal{X}^{b d d}\left(E_{i}\right)$ of bounded sections of $E_{i}$. Therefore, there exists a unique $v_{i} \in \mathcal{X}^{b d d}\left(E_{i}\right)$ such that $P_{i} \alpha\left(\gamma_{i}\right)=v_{i}-\left(\tilde{\rho}\left(\gamma_{i}\right)\right)_{*} v_{i}$. Since $P_{i} \alpha\left(\gamma_{i}\right)$ is continuous, $v_{i}$ is continuous.

On the other hand, if we define $v_{i}^{\prime} \in \mathcal{X}^{b d d}\left(E_{i}\right)$ by $v_{i}^{\prime}(x)=v_{i}(x)$ if $x \notin$ $O(p), v_{i}^{\prime}(x)=P_{i}\left(v_{0}(x)\right)$ if $x \in O(p)$, it is clear that $v_{i}^{\prime}$ also satisfies $P_{i} \alpha\left(\gamma_{i}\right)=$ $v_{i}^{\prime}-\left(\tilde{\rho}\left(\gamma_{i}\right)\right)_{*} v_{i}^{\prime}$. Hence $v_{i}=v_{i}^{\prime}$. In other words, continuous section $v_{i}$ extends set function $P_{i}\left(v_{0}\right)$ defined on a dense set $O(p)$. Corollary 2.7 ensures that $v_{0}$ extends to a continuous section $v$ which is clearly a $C^{0}$-coboundary for $\alpha$.

\subsection{Uniqueness and continuity of measurable coboundary.}

We insist in this subsection that a measurable section $v$ of $E \rightarrow M$ is essentially finite; i.e., for a continuous fiber norm $\|\cdot\|$ on the vector bundle $E \rightarrow M,\{x \in M:\|v(x)\|=\infty\}$ has zero Lebesgue measure. We remark that we do not assume the density of periodic points for $\rho$ in this subsection.

Proposition 3.3. Let $E=M \times \mathbb{R}^{n_{0}}$ and $\tilde{\rho}$ be a flat action of $\Gamma$ on $E$ associated with homomorphism $\tilde{\pi}$ without compact part, and $\tilde{\rho}$ covers an action $\rho$ of $\Gamma$ on $M$. Let $\rho$ preserve Lebesgue measure. Then for any map $\alpha$ from $\Gamma$ to measurable section of vector bundle $E \rightarrow M$, the equation $\alpha(\gamma)=v-(\tilde{\rho}(\gamma))_{*} v($ for all $\gamma \in \Gamma)$ has at most one measurable solution $v$.

Proof. Otherwise, there exists a nontrivial measurable section $u$, such that $u=(\tilde{\rho}(\gamma))_{*} u$ for all $\gamma \in \Gamma$. We fix a trivialization for $E$, and consider $u$ as a measurable map from $M$ to $\mathbb{R}^{n_{0}}$. Take $s, \gamma_{i}, E_{i}, F_{i}$ as in Lemma 2.6', $P_{i}$ as in Lemma 2.7. We claim that $u_{i}:=P_{i} u=0$ almost everywhere, for all $i=1, \ldots, s$. Indeed, for any $i=1, \ldots, s, u_{i}=\left(\tilde{\rho}\left(\gamma_{i}\right)\right)_{*} u_{i}$, or equivalently $u_{i}\left(\rho\left(\gamma_{i}\right) x\right)=\tilde{\pi}\left(\gamma_{i}\right) u_{i}(x)$, hence $u_{i}\left(\rho\left(\gamma_{i}^{n}\right) x\right)=\tilde{\pi}\left(\gamma_{i}^{n}\right) u_{i}(x)$ for all $n \geq 1$. Then Poincaré recurrence theorem implies that for almost all $x \in\{x \in M: u(x) \neq$ $0\},\|u(x)\|=\infty$. Contrary to the fact that $\{x \in M:\|u(x)\|=\infty\}$ has zero Lebesgue measure. 
This is the uniqueness results of the measurable coboundary (if exists) of cocycle $\alpha$. We remark that the following is proved above. Let $w_{i}$ be a measurable section of the vector bundle associated with $E_{i}$. Then there exists at most one measurable section $v_{i}$ of the vector bundle associated with $E_{i}$ satisfying $w_{i}=v_{i}-\left(\tilde{\rho}\left(\gamma_{i}\right)\right)_{*} v_{i}$. Based on this observation, we obtain the following continuity result of the measurable coboundary (if exists) of cocycle $\alpha$.

Proposition 3.4. Assume the conditions in Proposition 3.3. Let $\alpha$ be a continuous cocycle of $\Gamma$ with coefficient in $E$. Let $v$ be a measurable coboundary. Then $v$ coincides with a continuous section. Consequently, such measurable coboundary $v$ is unique.

Proof. Assume the notation as in the proof of Proposition 3.2. By the same argument as in the proof of Proposition 3.2, we know that $P_{i} \alpha\left(\gamma_{i}\right)=$ $v_{i}-\left(\tilde{\rho}\left(\gamma_{i}\right)\right) v_{i}$ has a unique continuous solution $v_{i}$. By the uniqueness result Proposition 3.3 and the remark above, $P_{i} v$ coincides with $v_{i}$. Our result follows from Corollary 2.7 .

\section{3. $L^{2}, C^{0}$ vanishing of $C^{r}$-cocycle for other actions.}

In this subsection, we always assume that $\tilde{\rho}$ is a smooth flat action of $\Gamma$ on a flat bundle $E=M \times \mathbb{R}^{n_{0}}$ associated with a homomorphism $\pi: \Gamma \rightarrow \mathbb{R}^{n_{0}}$ such that $\tilde{\rho}$ covers a smooth action $\rho$ of $\Gamma$ on $M$. Moreover $\pi$ decomposes as a direct sum of two subrepresentations $\pi=\pi_{1}+\pi_{2}$ corresponding to $\mathbb{R}^{n_{0}}=\mathbb{R}^{n_{1}} \oplus \mathbb{R}^{n_{2}}=W_{1} \oplus W_{2}$ where $\pi_{1}$ has no compact part and $\pi_{2}$ has only the compact part.

Proposition 3.5. Let $\Gamma, E, \tilde{\rho}, \rho$ and $\pi$ be as above. Assume that $\Gamma$ is an SVC and Kazhdan group, and that $\rho$ has dense periodic points.

1. If $\rho$ preserves Lebesgue measure, then any $C^{r}$-cocycle $\alpha$ is an $L^{2}$ coboundary; i.e., there exists $v \in \mathcal{X}^{L^{2}}(E)$, such that $\alpha(\gamma)(x)=$ $v-(\tilde{\rho}(\gamma))_{*} v(x)$ for all $\gamma \in \Gamma$ and almost all $x \in M$. Moreover, the projection $P_{1}(v)$ of $v$ to the first factor is continuous; for any two such coboundaries $u, v, P_{1}(u-v)$ is trivial, and the norm of the projection $P_{2}(u-v)$ of $u-v$ to the second factor is invariant under the action.

2. If $M$ is a torus or a nilmanifold, $\rho$ is an Anosov action by toral or nilmanifold automorphisms, and $\pi_{2}$ is trivial (i.e., $\pi_{2}(\gamma)=I d_{W_{2}}$ ). Then 
any $C^{r}$-cocycle $\alpha$ is a $C^{0}$-coboundary; i.e., there exists $v \in \mathcal{X}^{0}(E)$, such that $\alpha(\gamma)(x)=v-(\tilde{\rho}(\gamma))_{*} v(x)$ for all $\gamma \in \Gamma$ and all $x \in M$. Moreover, $P_{1}(v)$ is unique and continuous; $P_{2}(v)$ is of class $C^{\infty}$ if $r=\infty$; for any two such coboundaries $u, v, P_{1}(u-v)$ is trivial and $P_{2}(u-v)$ is constant.

Proof. We decompose $\mathbb{R}^{n_{0}}$ into the direct sum of $\pi$-invariant subspaces $W_{1} \oplus W_{2}$, so that $\left.\pi\right|_{W_{1}}$ has no compact part and $\left.\pi\right|_{W_{2}}$ has only compact part. We denote the corresponding $\tilde{\rho}$ smooth invariant subbundles of $E$ by $E_{1}$ and $E_{2}$. We decompose the cocycle $\alpha=\alpha_{1}+\alpha_{2}$ so that $\alpha_{i}$ is a $C^{r}$-cocycle with coefficients in $E_{i}$.

(1) The restriction $\tilde{\rho}_{1}$ of $\tilde{\rho}$ on flat bundle $E_{1}$ is a flat action of $\Gamma$ on flat bundle $E_{1}$ associated with the homomorphism $\pi_{1}$ with no compact part. So there exists unique $v_{1} \in \mathcal{X}^{0}\left(E_{1}\right)$ such that $\alpha_{1}(\gamma)=v_{1}-\left(\tilde{\rho}_{1}(\gamma)\right)_{*} v_{1}$ by Proposition 3.2. The induced action $\left(\tilde{\rho}_{2}\right)_{*}$ of the restriction $\tilde{\rho}_{2}$ of $\tilde{\rho}$ on flat bundle $E_{2}$ is a unitary action of $\Gamma$ on $\mathcal{X}^{L^{2}}\left(E_{2}\right)$, therefore there exists $v_{2} \in \mathcal{X}^{L^{2}}\left(E_{2}\right)$ such that $\alpha_{2}(\gamma)=v_{2}-\left(\tilde{\rho}_{2}(\gamma)\right)_{*} v_{2}$ by Kazhdan Property T. Other claims are easy to see. Our result follows.

(2) We only need to prove that $L^{2}$ section $v_{2}$ satisfying $\alpha_{2}(\gamma)=v_{2}-$ $\left(\tilde{\rho}_{2}(\gamma)\right)_{*} v_{2}$ is actually $C^{\infty}$ if $r=\infty$. Without loss of generality, we assume that $v_{2}$ is a real valued function. Fix an Anosov element $\gamma \in \Gamma$; i.e., $f=\rho(\gamma)$ is an Anosov automorphism. Our result follows from Theorem 2.3.

\subsection{Differential operator and induced actions.}

Assume that the tangent bundle of $M$ and the vector bundle $E \rightarrow M$ are flat (i.e., $T M=M \times \mathbb{R}^{n}, E=M \times \mathbb{R}^{n_{0}}$ ). Let $j^{1} E=L(T M, E)=$ $\cup_{x \in M} M_{n, n_{0}}(\mathbb{R})$, where $M_{n, n_{0}}(\mathbb{R})$ are the set of all $n \times n_{0}$ real-matrices. Let $f$ be a $C^{r}$-section of $E$, or equivalently, $f$ is a $C^{r}$-map from $M$ to $\mathbb{R}^{n_{0}}$. We assign $f$ to a $C^{r-1}$-section of $j^{1} E$, or equivalently a $C^{r-1}$ map, by $x \rightarrow D f(x)$. This assignment defines a differential operator $D: \mathcal{X}^{r}(E) \rightarrow \mathcal{X}^{r-1}\left(j^{1} E\right)$. Let $\tilde{\rho}$ be a flat action of discrete group $\Gamma$ on $E$ via homomorphism $\pi_{E}: \Gamma \rightarrow G L\left(n_{0}, \mathbb{R}\right)$. We define a flat action of $\Gamma$ on $j^{1} E$ as follows. Let $\gamma \in \Gamma$, and $w \in\left(j^{1} E\right)_{x}$. We may consider $w \in M_{n, n_{0}}$. Then $\gamma w \in\left(j^{1} E\right)_{\rho(\gamma) x}$, and as a matrix $\gamma w=\pi_{E}(\gamma) w$. This in turn induces an action of $\Gamma$ on $\mathcal{X}^{r}\left(j^{1}(E)\right)$ by $(\gamma u)(x)=\pi_{E}(\gamma) u\left(\rho\left(\gamma^{-1}\right) x\right)$.

If $\rho$ is tangentially flat, $\tilde{\rho}$ as above, then the action of $\Gamma$ on $j^{1} E$ is also flat. Denote by $\pi_{j^{1} E}$ the homomorphism associated with the flat action. From 
the construction of the actions we see that $D$ and the actions commute. Apply previous results in this section to this special bundle, we obtain the following result.

Proposition 3.6. Let $\rho$ be a tangentially flat action of $\Gamma$ on $M, \tilde{\rho}$ be a flat bundle action of $\Gamma$ on a flat bundle $E \rightarrow M$.

1. If $\alpha$ is a $C^{r}$-cocycle with coefficients in $E$, then $D \alpha$ is a $C^{r-1}$-cocycle with coefficients in $j^{1} E$;

2. If $\rho$ has dense periodic points and $\Gamma$ is SVC and Kazhdan, $\pi_{j^{1} E}$ decomposes into $\pi_{1}^{\prime}+\pi_{2}^{\prime}$ such that $\pi_{1}^{\prime}$ has no compact part and $\pi_{2}^{\prime}(\gamma)$ has only compact part. Then for every $C^{1}$-cocycle $\alpha, D \alpha$ is $L^{2}$ trivial;

3. If in addition to the assumptions in (2), we assume that $\rho$ is Anosov and $\pi_{2}^{\prime}(\gamma)$ is identity for all $\gamma \in \Gamma$. Then $D \alpha$ is $C^{0}$ trivial.

Proof. It follows from the construction above and Proposition 3.5. We mention that a tangentially flat action always preserves a Lebesgue measure (Lemma 2.5 (2) of [18]).

\section{4. $C^{\infty}$-infinitesimal rigidity.}

We assume in this section that $\Gamma$ is a discrete, Kazhdan, SVC group, $T M=M \times \mathbb{R}^{n}, \rho$ a tangentially flat action of $\Gamma$ on $M$ associated with a completely reducible homomorphism $\pi: \Gamma \rightarrow G L(n, \mathbb{R}), \alpha$ a $C^{\infty}$-cocycle with coefficients in TM, $v$ a $C^{0}$-coboundary of $\alpha$. If $M$ is a torus, we let $\rho$ be such that the linearizing framing can be taken as $\sigma=\left\{\frac{\partial}{\partial x_{1}}, \ldots, \frac{\partial}{\partial x_{n}}\right\}$; if $M$ is a quotient $H / \Lambda$, we let $\rho$ be such that the linearizing framing can be lifted to a framing of left invariant vector fields on $N$. We shall give sufficient conditions under which $v$ is of class $C^{\infty}$.

\subsection{Anosov actions.}

We first prove an algebraic lemma.

Lemma 4.1. Let $\mathcal{B}$ be a finitely generated abelian group, $\operatorname{Tor}(\mathcal{B})$ the torsion group of $\mathcal{B}$. Assume that the finitely generated free abelian group $\mathcal{B} / \operatorname{Tor}(\mathcal{B})$ has rank at least 1 , and that $\lambda, \mu: \mathcal{B} \rightarrow \mathbb{R}$ are two non-trivial homomorphisms. Then 
1. either there exists a positive number $c>0$ such that $\lambda(b)=c \mu(b)$ for all $b \in \mathcal{B}$;

2. or there exists $b \in \mathcal{B}$ such that $\lambda(b)<0$ and $\mu(b)>0$.

Proof. Let $B=\mathbb{Z}^{r} \oplus \operatorname{Tor}(\mathcal{B})$. Then it is easy to see that $\left.\lambda\right|_{\operatorname{Tor}(\mathcal{B})},\left.\mu\right|_{\operatorname{Tor}(\mathcal{B})}$ are trivial. So without loss of generality, we assume that $\mathcal{B}=\mathbb{Z}^{r} . \lambda, \mu$ can be extended to homomorphisms $\mathbb{R}^{r} \rightarrow \mathbb{R}$. We denote the extensions by $\lambda^{\prime}, \mu^{\prime}$, respectively.

Consider the kernels of $\lambda^{\prime}$ and $\mu^{\prime}$, we have either (i) $\operatorname{ker}\left(\lambda^{\prime}\right)=\operatorname{ker}\left(\mu^{\prime}\right)$ (denote the common space by $K$ ) or (ii) $\operatorname{ker}\left(\lambda^{\prime}\right) \neq \operatorname{ker}\left(\mu^{\prime}\right)$. If (i) occurs, take $b \in \mathbb{R}^{r}$ such that $\lambda^{\prime}(b), \mu^{\prime}(b) \neq 0$ and let $c=\lambda^{\prime}(b) / \mu^{\prime}(b)$. Then for any $a \in \mathbb{R}^{r}, a=k+h b$ for some $k \in K$ and $h \in \mathbb{R}$. So $\lambda^{\prime}(a)=h \lambda^{\prime}(b)$, $\mu^{\prime}(a)=h \mu^{\prime}(b)$. Hence $\lambda^{\prime}(a)=c \mu^{\prime}(a)$. If $c<0$ we have (2) as asserted in the lemma, if $c>0$ we have (1) as asserted in the lemma.

If (ii) occurs, then the negative half space of $\lambda^{\prime} N_{\lambda^{\prime}}:=\left\{u \in \mathbb{R}^{r}: \lambda^{\prime}(u)<\right.$ $0\}$ and the positive half space of $\mu^{\prime} P_{\mu^{\prime}}:=\left\{u \in \mathbb{R}^{r}: \mu^{\prime}(u)>0\right\}$ intersect in an open, non-empty cone $C \subset \mathbb{R}^{r}$. It is obvious that $\mathbb{Z}^{r} \cap C$ is non-trivial. Take any $b \in C$, we have (2) as asserted in the lemma.

Theorem 4.2. Let $\Gamma, \rho, \pi, \alpha$ and $v$ be as in the beginning of this section. Assume that $M$ is a torus or a nilmanifold, $\rho$ is an Anosov action by automorphisms, and $\pi$ is of almost Lyapunov multiplicity free type. Then $v$ is of class $C^{\infty}$.

Proof. It is clear that the action $\rho$ has dense periodic points in $M$. Let $\sigma$ be the linearizing framing. Let $V \subset \mathbb{R}^{n}$ be an irreducible $\pi$-invariant subspace, $\mathcal{A} \subset \Gamma$ an abelian subgroup, $\lambda$ a real multiplicity free Lyapunov exponent so that $E_{\lambda}^{\prime}:=E_{\lambda} \cap V \neq\{0\}$. Let the dimensions of $E_{\lambda}^{\prime}, E_{\lambda}$ be $s_{1}, s$, respectively. Denote the subbundle corresponding to $E_{\lambda}^{\prime}, E_{\lambda}$ by $W_{\lambda}^{\prime}, W_{\lambda}$. Without loss of generality, we assume that $\sigma=\left\{X_{1}, \ldots, X_{s_{1}}, X_{s_{1}+1}, \ldots, X_{s}, Y_{1}, \ldots, Y_{t}\right\}$ with $s+t=n, X_{i} C^{\infty}$-sections of $W_{\lambda}$ (among them $X_{1}, \ldots, X_{s_{1}}$ are sections of $\left.W_{\lambda}^{\prime}\right), Y_{i} C^{\infty}$-sections of subbundles corresponding to the other Lyapunov subspaces.

Since $\lambda$ is real Lyapunov exponent, $E_{\lambda}$ is the common eigenspace of $\mathcal{A}$ with eigenvalues given by $\exp (\lambda)$. By the tangential flatness of $\rho$, for $\gamma \in \mathcal{A}$ the equation $\alpha(\gamma)=v-(\rho(\gamma))_{*} v$ can be written as $\alpha(\gamma)(x)=$ $v(x)-\pi(\gamma) v\left(\rho\left(\gamma^{-1}\right) x\right)$. Let the $X_{1}$-component of $\alpha(\gamma)(x)$ be $\alpha_{1}(\gamma)(x)$, the $X_{1}$-component of $v(x)$ be $\phi(x)$. The $X_{1}$-component of $\pi(\gamma) v\left(\rho\left(\gamma^{-1}\right) x\right)$ is 
then $\exp (\lambda(\gamma)) \phi\left(\rho\left(\gamma^{-1}\right) x\right)$. Compare the $X_{1}$ components at both sides of the equation, we obtain

$$
\alpha_{1}(\gamma)(x)=\phi(x)-\exp (\lambda(\gamma)) \phi\left(\rho\left(\gamma^{-1}\right) x\right)
$$

Take an element $\gamma \in \mathcal{A}$ so that $\lambda(\gamma)<0$. Let $f(x)=\alpha_{1}(\gamma)(x), T=\rho(\gamma)$, then (4.1) has an explicit solution

$$
4.2 \phi(x)=f(x)+\exp (\lambda(\gamma)) f\left(T^{-1} x\right)+\cdots+\exp (\lambda(\gamma))^{k} f\left(T^{-k} x\right)+\ldots
$$

Take any $Y_{i}$, we want to show that for any positive integer $m, Y_{i}^{m} \phi(x)$ exist and are continuous, $i=1, \ldots, t$. Let $\mu$ be a Lyapunov exponent, $E_{\mu}$ the corresponding Lyapunov space. Let $W_{\mu}$ be the invariant subbundle corresponding to $\mu$ so that $Y_{i}$ is a vector field in the $W_{\mu}$. Since $\lambda$ is multiplicity free, there exists element $a \in \mathcal{A}$ such that $\exp (\lambda(a))<1, \exp (\mu(a))>1$ and $\rho(a)$ is Anosov. We take $\gamma=a$ in (4.1). It is easy to see that the formal derivative of the series with respect to $Y_{i}^{m}$ exist and converges uniformly for all $m$, therefore $Y_{i}^{m} \phi(x)$ exist and are continuous for all $m \geq 0$.

It remains to show that $X_{i}^{m} \phi(x)$ exist and continuous. Formally differentiate (4.1), we get a formal equation $X_{i} f(x)=X_{i} \phi(x)-X_{i} \phi\left(\rho\left(a^{-1}\right) x\right)$. Since equation $X_{i} f(x)=g_{i}(x)-g_{i}\left(\rho\left(a^{-1}\right) x\right)$ has an $L^{2}$-solution $g_{i}(x)$ by Proposition $3.6(2)$, and $\rho\left(a^{-1}\right)$ is Anosov, $g_{i}(x)$ is of class $C^{\infty}$ by Theorem 2.3. We recall a Hurder's trick [5]. The partial sum $\phi_{n}(x)$ of the first $n$ terms in the right hand side of (4.2) has derivative $X_{i} \phi_{n}(x)=$ $X_{i} f(x)+X_{i} f\left(T^{-1} x\right)+\cdots+f\left(T^{-(n-1)} x\right)=g_{i}(x)-g_{i}\left(T^{-n} x\right)$, and hence uniformly bounded. Therefore, $\phi(x)$ is absolutely continuous along the integral curves of $X_{i}$ and $X_{i} \phi(x)$ exist almost everywhere and $X_{i} \phi(x)$ is $L^{1}$. By the ergodicity of $\rho\left(a^{-1}\right)$, we have the uniqueness of the measurable coboundary, or $X_{i} \phi(x)=g_{i}(x)+c$ for a constant $c$. Hence $X_{i} \phi(x)$ is of class $C^{\infty}$.

We now apply a regularity theorem (Theorem 2.1 of [6]), we see that $\phi(x)$, the $X_{1}$-component of $v(x)$, is of class $C^{\infty}$. Same argument shows that for any $X_{j}, X_{j}$-component of $v(x)$ is of class $C^{\infty}$.

In other words, in term of notation in Theorem 2.9, we may decompose $\mathbb{R}^{n}=E(a) \oplus F(a)$ into a direct sum of the sum of some subsets of generalized eigenspaces of $\pi(a)$ with $E(a)$ generated by $X_{1}, \ldots, X_{s}$ and $F(a)$ generated by $Y_{1}, \ldots, Y_{t}$, so that $v_{(E(a), F(a))}$ is of class $C^{\infty}$. For any $\gamma^{\prime} \in \Gamma$, we replace $\mathcal{A}$ by $\gamma^{\prime} \mathcal{A} \gamma^{\prime-1}$, replace $a$ by $\gamma^{\prime} a \gamma^{\prime-1}$, replace $E(a)$ and $F(a)$ by $E\left(\gamma^{\prime} a \gamma^{-1}\right)$ and $F\left(\gamma^{\prime} a \gamma^{\prime-1}\right)$ respectively, repeat the process above. We conclude that $v_{\left(E\left(\gamma^{\prime} a \gamma^{\prime-1}\right), F\left(\gamma^{\prime} a \gamma^{\prime-1}\right)\right)}$ is also of class $C^{\infty}$.

Applying Theorem 2.9, we obtain that $v(x)$ is itself of class $C^{\infty}$. 
For Anosov action $\rho$ of almost Lyapunov exponent free type, the proof above goes through with minor changes. We leave the proof as an exercise.

\subsection{Partially hyperbolic actions.}

The first examples of partially hyperbolic, $C^{\infty}$-infinitesimal actions are the product actions. To be specific, we have the following.

Theorem 4.3. let $M$ be a torus or a nilmanifold, $\rho_{0}$ an Anosov action of $\Gamma$ on $M$ by automorphisms. Let $\Gamma$ be a SVC and Kazhdan group, and $\rho_{0} C^{\infty}$-infinitesimal rigid. Then the product action $\rho$ of $\Gamma$ on $M \times N$ by $\rho(\gamma)(m, n)=\left(\rho_{0}(\gamma) m, n\right)$, where $N$ is a connected smooth manifold but not necessarily compact, is also $C^{\infty}$-infinitesimal rigid.

To prove the theorem, we need two simple lemmas.

Lemma 4.4. Let $M, N$ be as in the theorem. Let $T: M \rightarrow M$ be an Anosov automorphism. Let $f: M \times N \rightarrow \mathbb{R}$ be $C^{\infty}$ and $\phi: M \times N \rightarrow$ $\mathbb{R}$ be $L^{2}$ such that $f(m, n)=\phi(m, n)-\phi(T m, n)$. Then there exists $C^{\infty}$ function $\phi_{\infty}: M \times N \rightarrow \mathbb{R}$ such that $f(m, n)=\phi_{\infty}(m, n)-\phi_{\infty}(T m, n)$ and $\phi_{\infty}(m, n)-\phi(m, n)=\eta(n)$ is $L^{2}$ and independent of $m$, for all $(m, n) \in$ $M \times N$.

Proof. For fixed $n \in N, \phi(m, n)$ is a $C^{\infty}$-function on $M$ by Theorem 2.3. Now for any given $m_{0} \in M$, any real valued $C^{\infty}$-function $\psi(n)$, there exists $C^{\infty}$-function $\phi_{\infty}$ so that $f(m, n)=\phi_{\infty}(m, n)-\phi_{\infty}(T m, n)$ and $\phi_{\infty}\left(m_{0}, n\right)=$ $\psi(n)$ by Proposition 2.4. It is easy to see that $\phi_{\infty}(m, n)-\phi(m, n)=\eta(n)$ is $L^{2}$ and independent of $m$, for all $(m, n) \in M \times N$.

Lemma 4.5. Let $\rho_{0}$ be a tangentially flat Anosov action of a SVC and Kazhdan group $\Gamma$ on $M$, associated with a homomorphism $\pi$. Let $\Gamma$ be such that every finite dimensional representation of it decomposes into two subrepresentations, one has no compact part, the other is identity. Assume that $\rho_{0}$ has dense periodic points and is $C^{\infty}$-infinitesimal rigid. Let $N$ be a connected smooth manifold (not necessarily compact), $\rho$ be an action of $\Gamma$ on $M \times N$ by $\rho(\gamma)(m, n)=\left(\rho_{0}(\gamma), n\right), \alpha$ be a $C^{\infty}$-cocycle over $\rho$ with coefficient in TM. Then there exists a unique $L^{2}$-coboundary $v$ such that

1. for fixed $n, v(\cdot, n)$ is a $C^{\infty}$-section of $T M$; 
2. for any $C^{\infty}$-vector field $X$ that is a vector field (on $M$ ) in the linearizing framing, $X^{k} v$ is a locally $L^{2}$-function on $M \times N$ for any $k \geq 1$;

3. for any $C^{\infty}$-vector field $Y$ on $N, Y^{k} v$ is a $C^{0}$-function on $M \times N$ for any $k \geq 1$.

4. $v$ is of class $C^{\infty}$.

Proof. The existence of $L^{2}$-coboundary $v$ can be derived from Proposition 3.5 (1). (Note we may take a Lebesgue measure on $N$ so that the volume of $N$ is finite and the arguments there is valid.)

(1) is the consequence of the $C^{\infty}$-infinitesimal rigidity of $\rho_{0}$.

(2) $X^{k} v$ is measurable by (1). Consider $D=D_{M}$ as a partial differential operator on $T M \times T N$, by repeated use of Proposition 3.6, we see that $D^{k} \alpha$ has an $L^{2}$-coboundary $g$. Let the induced bundle be $j^{k} T M:=$ $j^{1}\left(j^{(k-1)} T M\right)$, and the associated representation $\pi^{k}=\left(\pi^{k}\right)_{1}+\left(\pi^{k}\right)_{2}\left(\left(\pi^{k}\right)_{1}\right.$ has no compact part, $\left(\pi^{k}\right)_{2}$ has only compact part, the corresponding spaces of $\left(\pi^{k}\right)_{1}$ and $\left(\pi^{k}\right)_{2}$ are $U_{1}, U_{2}$ respectively). Since $D^{k} v$ is easily seen to be a measurable coboundary of $D^{k} \alpha$, we see that the $U_{1}$ component of $D^{k} v-g$ is trivial and the the $U_{2}$ component of $D^{k} v-g$ is invariant under the action.

By looking at the component of $D v-g$ with the above consideration, we conclude that $X v=g_{1}+f_{1}$ where $g_{1}$ is $L^{2}$ and $f_{1}$ is measurable and invariant under the action. Hence $f_{1}=f_{1}(n)$. By integration along the trajectories of $X$, we see that $f_{1}(n)$ is locally $L^{2}$. Hence $X v$ is locally $L^{2}$. Similar argument yields that $X^{k} v$ is locally $L^{2}$.

(3) can be shown as follows. For Anosov element $\gamma \in \Gamma$ for $\rho_{0}$, we decompose $\mathbb{R}^{n}$ into stable and unstable subspaces of $\pi(\gamma)$. We decomposes $T M=E^{-} \oplus E^{+}$into stable subbundle and unstable subbundle of $T \rho_{0}(\gamma)$. We let $\alpha_{-}, \alpha_{+}, v_{-}, v_{+}$be the components of $\alpha, v$ in the obvious sense. Then the equation $\alpha(\gamma)(m, n)=v(m, n)-\pi(\gamma) v\left(\rho_{0}\left(\gamma^{-1}\right) m, n\right)$ decomposes into $\alpha_{ \pm}(\gamma)(m, n)=v_{ \pm}(m, n)-\pi(\gamma) v_{ \pm}\left(\rho_{0}\left(\gamma^{-1}\right) m, n\right)$. Each equation has an explicit absolutely and uniformly convergent series solution (on any compact subset of $N$ ). Moreover, after applying $Y$ to each of the terms of the series, we again obtain an absolutely and uniformly convergent series solution (on any compact subset of $N$ ). Hence $Y^{k} v_{ \pm}$are $C^{0}$ for any $k \geq 1$ and our result follows.

(4) It is a straightforward application of Theorem 2.1 of [6]. 
Proof. of Theorem 4.3. Take any $C^{\infty}$-cocycle $\alpha$ with coefficients in $T(M \times N)=T M \times T N$, we decompose $\alpha=\alpha_{M}+\alpha_{N}$. We have that $\alpha_{M}$ is a $C^{\infty}$-cocycle with coefficients in vector bundle $E_{M}=T M \times\{0\} \rightarrow M \times N, \alpha_{N}$ is a $C^{\infty}$-cocycle with coefficients in vector bundle $E_{N}=\{0\} \times T N \rightarrow M \times N$. The action $\rho$ induces a flat action $\rho^{M}$ of $\Gamma$ on $E_{M}$ associated with a homomorphism $\pi$ without compact factor ( $\rho_{0}$ is Anosov), a flat action $\rho^{N}$ of $\Gamma$ on $E_{N}$ associated with the trivial homomorphism. Since $\rho$ has dense periodic points, there exists $v_{M} \in \mathcal{X}^{0}\left(E_{M}\right)$ such that $\alpha_{M}(\gamma)=v_{M}-\left(\rho_{*}^{M}(\gamma)\right) v_{M}$; or (using the linearizing framing) we have $\alpha_{M}(\gamma)(m, n)=v_{M}(m, n)-$ $\pi(\gamma) v_{M}\left(\rho_{0}\left(\gamma^{-1}\right) m, n\right)$. Since $\rho_{0}$ is $C^{\infty}$-infinitesimal rigid, $v_{M}(m, n)$ is $C^{\infty}$ by Lemma 4.5 .

By Kazhdan property, we see that there exists $v_{N} \in \mathcal{X}^{L^{2}}(E)$ such that $\alpha_{N}(\gamma)(m, n)=v_{N}(m, n)-v_{N}\left(\rho_{0}\left(\gamma^{-1}\right) m, n\right)$. Without loss of generality we assume that $v_{N}$ is a real valued function. To show that $v_{N}$ can be taken as a $C^{\infty}$-function, we fix an Anosov element $\gamma_{0} \in \Gamma$ for the action $\rho_{0}$, and denote $\rho_{0}\left(\gamma_{0}\right)$ by $T$. Then we have $\alpha_{N}\left(\gamma_{0}\right)(m, n)=v_{N}(m, n)-v_{N}(T m, n)$. By Lemma 4.4, there exists $C^{\infty}$ function $v_{\infty}$ such that $\alpha_{N}\left(\gamma_{0}\right)(m, n)=$ $v_{\infty}(m, n)-v_{\infty}(T m, n)$, and $v_{N}(m, n)-v_{\infty}(m, n)=\eta(n)$ for an $L^{2}$-function $\eta$. For any $\gamma \in \Gamma, v_{\infty}(m, n)-v_{\infty}\left(\rho_{0}(\gamma) m, n\right)=v_{N}(m, n)-v_{N}\left(\rho_{0}(\gamma) m, n\right)=$ $\alpha_{N}(\gamma)(m, n)$. So $v_{\infty}$ can be taken as a $C^{\infty}$-coboundary of $\alpha_{N}$.

Another class of examples is the tangentially flat partially hyperbolic action on torus so that the corresponding homomorphism $\pi$ has no compact part.

Theorem 4.6. Let $\Gamma$ be an SVC group, $\rho$ an action of $\Gamma$ on torus $\mathbb{T}^{n}$ induced by completely reducible homomorphism $\pi: \Gamma \rightarrow S L(n, \mathbb{Z})$ without compact part. Let $\pi$ be of almost Lyapunov multiplicity free type. Moreover, assume that there exists a partially hyperbolic element $\gamma \in \mathcal{A}$ such that all unit eigenvalues are 1 , and $\lambda(\gamma)<0(\mathcal{A}, \lambda$ as in the definition of almost Lyapunov multiplicity freeness). Then $v$ is of class $C^{\infty}$.

Proof. The argument of the proof of Theorem 4.2 applies here except one minor change. We will indicate the difference and handle it separately.

It is clear that the action $\rho$ has dense periodic points in $M$. As in the proof of Theorem 4.2, let $\sigma$ be the linearizing framing as above. Let $V \subset \mathbb{R}^{n}$ be an irreducible $\pi$-invariant subspace, $\mathcal{A} \subset \Gamma$ an abelian subgroup, $\lambda$ a real multiplicity free Lyapunov exponent so that $E_{\lambda}^{\prime}:=E_{\lambda} \cap V \neq\{0\}$. Let the dimensions of $E_{\lambda}^{\prime}, E_{\lambda}$ be $s_{1}, s$, respectively. Denote the subbundle 
corresponding to $E_{\lambda}^{\prime}, E_{\lambda}$ by $W_{\lambda}^{\prime}, W_{\lambda}$. Without loss of generality, we assume that $\sigma=\left\{X_{1}, \ldots, X_{s_{1}}, X_{s_{1}+1}, \ldots, X_{s}, Y_{1}, \ldots, Y_{t}\right\}$ with $s+t=n, X_{i} C^{\infty}$ sections of $W_{\lambda}$ (among them $X_{1}, \ldots, X_{s_{1}}$ are sections of $W_{\lambda}^{\prime}$ ), $Y_{i} C^{\infty}$-sections of subbundles corresponding to the other Lyapunov subspaces.

Take a $C^{\infty}$-cocycle $\alpha$ with coefficients in $T M$, let $v \in \mathcal{X}^{0}(T M)$ be a coboundary of $\alpha, \phi(x), \alpha_{1}$ be the $X_{1}$-components of $v, \alpha$, respectively. For simplicity, we assume that $\lambda$ is Lyapunov multiplicity free. Same argument as in the proof of Theorem 4.2, we obtain that for any $k \geq 1, Y_{i}^{k} \phi(x)$ is continuous.

It remains to show that $X_{i}^{m} \phi(x)$ exist and are continuous. Let $\gamma$ be as in the statement of the Theorem, so that $\lambda(\gamma)<0$. Since

$$
\alpha_{1}(\gamma)(x)=\phi(x)-\exp (\lambda(\gamma)) \phi\left(\rho\left(\gamma^{-1}\right) x\right)
$$

by formal differentiation and let $f(x)=\alpha_{1}(\gamma)(x)$, we get equation $X_{i} f(x)=$ $X_{i} \phi(x)-X_{i} \phi\left(\rho\left(\gamma^{-1}\right) x\right)$. Since equation $X_{i} f(x)=g_{i}(x)-g_{i}\left(\rho\left(\gamma^{-1}\right) x\right)$ has an $L^{2}$-solution $g_{i}(x)$ by Proposition $3.6(2)$, and $T:=\rho\left(\gamma^{-1}\right)$ can be considered as a diffeomorphism on $\mathbb{T}^{n_{1}} \times \mathbb{T}^{n-n_{1}}$ by Anosov diffeomorphism on (torus) the first factor $M=\mathbb{T}^{n_{1}}$, by identity on the second factor $N=\mathbb{T}^{n-n_{1}}$, there exists $C^{\infty}$ function $g_{\infty}: M \times N \rightarrow \mathbb{R}$ such that $\alpha(\gamma)(m, n)=g_{\infty}(m, n)-$ $g_{\infty}(T m, n)$ and $g_{\infty}(m, n)-g(m, n)=\eta(n)$ is $L^{2}$ and independent of $m$, for all $(m, n) \in M \times N$ (Lemma 4.4). Therefore, $X_{j}^{k} g_{i}(x)$ is of class $C^{\infty}$ for any $k \geq 1$ and $j$ (we note that $X_{j}$ is tangent to the first factor $M$ ).

Since (4.2) has a series solution, Hurder's trick (see the proof of Theorem 4.2) implies that $\phi(x)$ is absolutely continuous along the integral curves of $X_{i}$ and $X_{i} \phi(x)$ exist almost everywhere and $X_{i} \phi(x)$ is $L^{1}$. By the ergodicity of $T$ on the first factor $M$, we have $X_{i} \phi(m, n)=g_{\infty}(m, n)+c(n)$ for an $L^{1}$-function $c$. By integration along trajectories of $X_{i}$, we see that $c(n)$ is also an $L^{2}$-function. Hence $X_{i}^{k} \phi(m, n)$ is of class $L^{2}$ for any $k \geq 1$.

We now apply a regularity theorem (Theorem 2.1 of [6]), we see that $\phi(x)$, the $X_{1}$-component of $v(x)$, is of class $C^{\infty}$. Apply the same argument as in the proof Theorem 4.2, we obtain that $v(x)$ is itself of class $C^{\infty}$.

Again for action $\rho$ of almost Lyapunov exponent free type, the proof above goes through with minor changes. We leave the proof as an exercise.

Still another class of partially hyperbolic $C^{\infty}$-infinitesimal actions will be considered in the next subsection. 


\subsection{Type 2 standard actions.}

We first recall the following result of Zimmer. We reformulate using our terminology.

Theorem 4.7. (Theorems 4.4-4.6 of [20]) Let $G$ be a connected semisimple Lie group with no simple factors locally isomorphic to $O(1, n)$ or $U(1, n)$, $\Gamma \subset G$ a cocompact lattice, $\pi: G \rightarrow G L\left(n_{0}, \mathbb{R}\right)$ a homomorphism. Let $\tilde{\rho}$ be a $C^{\infty}$ flat action of $\Gamma$ on flat bundle $E=M \times \mathbb{R}^{n_{0}}$ associated with $\pi$ covering a $C^{\infty}$-action $\rho$ preserving a smooth volume density. Then $\tilde{\rho}$ is $L^{2}$-infinitesimal rigid; i.e., any $C^{\infty}$-cocycle with coefficients in $E$ is a $L^{2}$-coboundary. In particular, let $H$ be a Lie group, $h: G \rightarrow H$ a continuous homomorphism, $\Lambda \subset H$ a cocompact lattice. Then the action of $\Gamma$ on $M=H / \Lambda$ is $L^{2}$ infinitesimal rigid $(E=T M)$.

Corollary 4.8. If $\pi$ does not contain the identity, then $\tilde{\rho}$ is $(\infty, 0)$ infinitesimal rigid; i.e., any $C^{\infty}$-cocycle with coefficients in $E$ is a $C^{0}$ coboundary.

Proof. Same argument as in the proof of Proposition 3.2.

While Zimmer gave conditions under which the type 2 actions are $C^{\infty}$ infinitesimal rigid using Sobolev space technique, we show the infinitesimal rigidity using harmonic analysis on semisimple Lie groups. The key ingredient is the use of the vanishing of the matrix coefficients for unitary representations. This approach was developed by Katok and Spatzier in establishing the cohomological and local rigidity of principal abelian group actions [6, 7], and then used by Kononenko for the cohomological rigidity of higher rank lattice actions [8]. They used the exponential decay of the matrix coefficients (Corollary 7.2 of [3]) for unitary representations to construct a distribution, and then show that the distribution is actually a smooth function.

We start with a $C^{\infty}$-cocycle $\alpha$ with coefficients in tangent bundle of $H / \Lambda$, and an $L^{2}$-coboundary $v$. Then we only need the vanishing of the matrix coefficients for unitary representations to construct a distribution. The regularity of the distribution can be established the same way as in $[6,7]$.

We recall a theorem on the vanishing of matrix coefficients.

Theorem 4.9. (By Howe-Moore, Sherman, and Zimmer, see Theorem 2.2.20 of [19]) Let $H$ be a finite product of connected non-compact sim- 
ple Lie groups $H_{i}$ with finite center, $U$ a unitary representation of $H$ on a Hilbert space $V$ such that for each $G_{i},\left.U\right|_{G_{i}}$ has no invariant vectors. Then for any $v, w \in V$, the matrix coefficients $\langle U(h) v, w>\rightarrow 0$ as $h$ leaves compact subsets of $H$.

Corollary 4.10. Let $H$ be as in Theorem 4.9, $\Lambda$ an irreducible cocompact lattice in $H$. Let $f_{1}, f_{2} \in L^{2}(H / \Lambda)$ orthogonal to constant functions (i.e., $\int f_{i} d x=0$, where $d x$ is a Haar measure on $\left.H / \Lambda, i=1,2\right)$. Then $\int \bar{f}_{1}(x) f_{2}(h x) d x \rightarrow 0$ as $h$ leaves compact subsets of $H$.

Proof. Let $U$ be the unitary representation of $H$ on $L^{2}(H / \Lambda)$ by $(U(h) f)(x)=f(h x)$. Then $U$ induces a unitary representation of $H$ on $V=L^{2}(H / \Lambda) \ominus \mathbb{C}$. Then Moore ergodic theorem asserts that there exist no non-trivial invariant vectors in $V$.

Theorem 4.11. Let $H$ be as in Theorem 4.9, $\Lambda$ an irreducible cocompact lattice in $H$. Let $\mathfrak{h}=\oplus \mathfrak{h}_{i}$ be the decomposition of the Lie algebra of $H$. Let $\rho$ be an action of a discrete group $\Gamma$ on $H / \Lambda$ via homomorphism $h: \Gamma \rightarrow H$. Assume that

1. $\rho$ is $L^{2}$-infinitesimal rigid;

2. $A d_{H} \circ h: \Gamma \rightarrow G L(\mathfrak{h})$ has no compact part;

3. there exists $\gamma_{0} \in \Gamma$ such that $A d_{H} \circ h\left(\gamma_{0}\right)$ is partially hyperbolic;

4. the eigenspace $V_{1} \subset \mathfrak{h}$ corresponding to eigenvalue 1 of $A d_{H} \circ h\left(\gamma_{0}\right)$ satisfies $V_{1} \cap \mathfrak{h}_{i} \neq \mathfrak{h}_{i}, \neq\{0\}$;

5. the only non-trivial irreducible invariant spaces of $A d_{H} \circ h$ are $\mathfrak{h}_{i}$.

Then $\rho$ is $C^{\infty}$-infinitesimal rigid.

Proof. $\rho$ is tangentially flat action of $\Gamma$ associated with homomorphism $A d_{H} \circ h$. For any $C^{\infty}$-cocycle with coefficients in $T M$, by (1) there exists an $L^{2}$-coboundary $v$ such that

$$
\alpha(\gamma)(x)=v(x)-A d_{H} \circ h(\gamma) v\left(h(\gamma)^{-1} x\right) .
$$

Since 1 is an eigenvalue of $A d_{H} \circ h\left(\gamma_{0}\right)$, and $V_{1}$ intersects each $\mathfrak{h}_{i}$ non-trivially, there exists a nonvanishing vector field (considered as an element in Lie 
algebra $\mathfrak{h}) X_{1} \in \mathfrak{h}_{i}$ on $H / \Lambda$ corresponding to eigenvalue 1 . Let $X_{2}, \ldots, X_{d}$ be complementary vector fields in the subbundle $E_{1}$ corresponding to Lyapunov exponent $0, Y_{1}, \ldots, Y_{k}$ be vector fields in the subbundle $E_{2}$ corresponding to other Lyapunov exponent of $A d_{H} \circ h\left(\gamma_{0}\right)$. We want to show that the $X_{1}$-component of $v$ is smooth.

Let $f, \phi$ be the $X_{1}$-components of $\alpha\left(\gamma_{0}\right), v$ respectively, Let $h\left(\gamma_{0}^{-1}\right)=g$. Compare the $X_{1}$-components of (4.3), we obtain $f(x)=\phi(x)-\phi(g x)$. It follows by repeated use of the equation that $\phi(x)=\sum_{i=0}^{m} f\left(g^{i} x\right)+\phi\left(g^{m+1} x\right)$ and $\phi(x)=-\sum_{i=1}^{m} f\left(g^{-i} x\right)+\phi\left(g^{-(m+1)} x\right)$. By the vanishing of the matrix coefficients (Theorem 4.9, Corollary 4.10) and (3), we obtain that as distribution, $P_{+}=\sum_{i=0}^{\infty} f\left(g^{i} x\right)$ and $P_{-}=-\sum_{i=1}^{\infty} f\left(g^{-i} x\right)$ are well-defined and they are equal. (We remark that $\int f(x) d x=\int(\phi(x)-\phi(g x)) d x=0$. If a function $u(x)$ is constant, then $\left.P_{+}(u)=P_{-}(u)=0\right)$.

We show that the stable and unstable spaces of $T:=A d_{H} \circ h\left(\gamma_{0}^{-1}\right)$ generate $\mathfrak{h}$. Since $T$ is an automorphism of $\mathfrak{h}$, we may decompose $\mathfrak{h}$ as $\mathfrak{h}=\mathfrak{h}^{0}+\mathfrak{h}^{+}+\mathfrak{h}^{-}$, where $\mathfrak{h}^{0}, \mathfrak{h}^{+}, \mathfrak{h}^{-}$are the sum of Lyapunov spaces corresponding to zero Lyapunov exponent, positive Lyapunov exponents and negative Lyapunov exponents of $T$ respectively. Since $\left[\mathfrak{h}^{0}, \mathfrak{h}^{+}\right] \subset \mathfrak{h}^{+}$, $\left[\mathfrak{h}^{0}, \mathfrak{h}^{-}\right] \subset \mathfrak{h}^{-}$, it is easy to obtain that the Lie algebra $\mathfrak{h}^{*}$ generated by $\mathfrak{h}^{+}, \mathfrak{h}^{-}$is a non-trivial idea. Hence $\mathfrak{h}^{*}$ is a sum of some $\mathfrak{h}_{i}$. If $\mathfrak{h}^{*} \neq \mathfrak{h}$, then some $\mathfrak{h}_{j} \subset \mathfrak{h}^{0}$ (since $\left\{\mathfrak{h}_{j}\right\}$ are permuted by $T$ and all of them are fixed by some positive power $T^{q}$ of $T$. If $\mathfrak{h}_{j}$ is not contained in $\mathfrak{h}^{*}$, and is invariant under $\left.T^{q}, \mathfrak{h}_{j} \subset \mathfrak{h}^{0}\right)$. Contrary to assumption (4).

Once $P_{+}=P_{-}$and the stable and unstable distributions of $g=h\left(\gamma_{0}^{-1}\right)$ generate $\mathfrak{h}$, Katok and Spatzier showed (see Theorem 4.5 of [7]) that $P=$ $P_{+}=P_{-}$is a $C^{\infty}$-function. Hence the $X_{1}$-component $\phi$ of $v$ is of class $C^{\infty}$.

Apply the same argument as in the proof of Theorem 4.2, we obtain that $v(x)$ is itself of class $C^{\infty}$. 


\section{References.}

[1] P.J. Fleming (1983), Structure stability and group cohomology, Trans. AMS Vol.275, No.2, 791-809.

[2] P. de la Harpe and A. Valette (1989), La propriété (T) de Kazhdan pour les groupes localement compacts, Astérisques, Soc. Math. de France, 175.

[3] R. Howe (1980), A notion of rank for unitary representations of the classical groups, A. Figà Talamanca (ed.), Harmonic analysis and group representations, CIME.

[4] S. Hurder (1992), Rigidity for Anosov actions of higher rank lattices, Jour. Ann. of Math, 135, 361-410.

[5] S. Hurder (1995), Infinitesimal rigidity for hyperbolic actions, J. Diff. Geom., Vol.41, No.3, 515-527.

[6] A. Katok and R. Spatzier(1994), Subelliptic estimates of polynomial differential operators and applications to rigidity of Abelian actions, Math. Res. Letters, 1, 193-202.

[7] A. Katok and R. Spatzier(1994), First cohomology of Anosov actions of higher rank abelian group and applications to rigidity, Publ. IHES, 79, 131-156.

[8] A. Kononenko (1994), Cohomological rigidity of actions of higher rank lattices, Preprint.

[9] J. Lewis(1991), Infinitesimal rigidity for the action of $S L\left(n, \mathbb{Z}^{n}\right)$ on $\mathbb{T}^{n}$, Trans. AMS, 324, 421-445.

[10] A. Livsic(1971), Homology properties of U systems,Math Notes, Vol.10, 758763.

[11] A. Livsic(1972), Cohomology of dynamical systems, Mthe. USSR Izvestija, Vol.6, No.6, 1278-1301.

[12] R. de la Llave, J.M. Marco and R. Moriyon (1986), Canonical perturbation theory of Anosov systems and regularity results for the Livsic cohomology equation, Ann. Math, 123, 537-611.

[13] A. Lubotzky and R. J. Zimmer (1989), Variants of Kazhdan's property for subgroups of semisimple groups, Israel J. Math., 66, 289-299.

[14] G. A. Margulis (1991), Discrete subgroups of semisimple Lie groups, SpringerVerlag, New York. 
[15] G. D. Mostow (1970), Intersections of discrete subgroups with Cartan subgroups, J. Indian Math. Soc., 34, 203-214.

[16] G. Prasad, $\mathbb{R}$-regular elements in Zariski-dense subgroups, to appear in the Oxford Quarterly J. of Math.

[17] G.Prasad and M.S.Raghunathan (1972), Cartan subgroups and lattices in semisimple groups, Ann. Math, 96, 296-317.

[18] N. Qian and R.J. Zimmer (1996), Entropy rigidity for semisimple group actions, to appear in Israel J. of Math.

[19] R. J. Zimmer (1986), Actions of semisimple groups and discrete subgroups, Proceedings of the International Congress of Mathematicians, Berkeley, 12471258.

[20] R. J. Zimmer (1990), Infinitesimal rigidity of discrete subgroups of Lie groups, J. Diff. Geom., 31, 301-322.

RECEIVEd MARCH 22, 1995.

DEPARTMENT OF MathEMATICS

YALE UNIVERSITY

NEW HAVEN, CT 06520

E-MAIL: QIAN@MATH.YALE.EDU 